\begin{tabular}{|c|c|}
\hline Title & Horizontal diffusive motion of columnar vortices in rotating Rayleigh-Benard convection \\
\hline Author(s) & Noto, D.; T asaka, Y .; Y anagisawa, T.; Murai, Y. \\
\hline Citation & $\begin{array}{l}\text { Journal of fluid mechanics, 871, 401-426 } \\
\text { https://doi.org/10.1017/Jfm.2019.313 }\end{array}$ \\
\hline Issue Date & 2019-07-25 \\
\hline DOC URL & http:/hdl.handle.net/2115/76593 \\
\hline Rights & $\begin{array}{l}\text { This article has been published in a revised form in Journal of fluid mechanics http://doi.org/10.1017 ffm.2019.313. } \\
\text { This version is free to view and download for private research and study only. Not for re distribution, re sale or use in } \\
\text { derivative works. @ } 2019 \text { Cambridge University Press }\end{array}$ \\
\hline Type & article (author version) \\
\hline File Information & rrbc_jfm_latex_ver12.pdf \\
\hline
\end{tabular}

Instructions for use 


\title{
Horizontal diffusive motion of columnar vortices in rotating Rayleigh-Bénard convection
}

\author{
D. Noto ${ }^{1}$, Y. Tasaka $^{1} \dagger$, T. Yanagisawa ${ }^{2,1}$ and Y. Murai ${ }^{1}$ \\ ${ }^{1}$ Laboratory for Flow Control, Faculty of Engineering, Hokkaido University, Sapporo, Japan \\ ${ }^{2}$ Japan Agency for Marine-Earth Science and Technology (JAMSTEC), Yokosuka, Japan
}

(Received xx; revised xx; accepted xx)

In laboratory experiments, horizontal translational motion of columnar vortices formed in rotating Rayleigh-Bénard convection was investigated. Two types of measurements, vertical velocity fields and horizontal temperature fields, were conducted with water as the test fluid. Using particle image velocimetry, the vertical velocity fields determined the parameter range at which the quasi-two-dimensional columnar vortices emerged. Locally, the duration characteristics of the columns, evaluated with their vertical coherence, indicate the minimum timescale of translational motion of the vortices in the horizontal plane. Vortex tracking of the horizontal temperature fields over long observation periods $>10^{3} \mathrm{~s}$, were obtained using encapsulated thermochromic liquid crystal visualization. Two cylindrical vessels with different radii showed the emergence of the centrifugal effect in $O\left(>10^{2} \mathrm{~s}\right)$ despite the small Froude number, $\mathrm{Fr}<0.1$. Further, fashion in the horizontal plane the columnar vortices behaved in a random-walk-like diffusive motion. The statistically calculated mean-squared displacements indicated anomalous diffusive motion of the columns; displacement increasing with time as $t^{\gamma}$ with $\gamma \neq 1$. We discuss the causes of this anomaly in both the instantaneous and long-term statistical data gathered from experimental observations over different timescales. The enclosure effect from the repulsion of up-welling and down-welling vortices ensure that vortices diffuse only little, resulting in a sub-diffusive (decelerated) motion $\gamma<1$ in $O\left(10^{1} \mathrm{~s}\right)$. With this weak centrifugal contribution, the translational motion of the columns slowly accelerates in the radial direction and thereby yields a super-diffusive (accelerated) motion $\gamma>1$ in $O\left(10^{2} \mathrm{~s}\right)$.

Key words: Authors should not enter keywords on the manuscript, as these must be chosen by the author during the online submission process and will then be added during the typesetting process (see http://journals.cambridge.org/data/relatedlink/jfmkeywords.pdf for the full list)

\section{Introduction}

Rayleigh-Bénard convection (RBC), the thermal convection driven between two horizontal plates at different temperatures, is a fundamental fluid mechanics system involving large-scale fluid motion. With the addition of a background rotation to RBC, a socalled rotating $\mathrm{RBC}$, has attracted great worldwide interests applied to geophysical and

$\dagger$ Email address for correspondence: tasaka@eng.hokudai.ac.jp 
astrophysical flows. Meteorological flows such as typhoons and tornadoes are examples of rotating RBC as the influence of the Earth's rotation here can then be understood intuitively (e.g., Meuel et al. 2012). In different scenarios, planetary magnetic fields are maintained by the convective motion of liquid metal influenced by the rotation in an outer core (Jones 2011; Grannan et al. 2016). For the physics of large length and time-scale systems, non-dimensional parameters such as Rayleigh and Taylor numbers would be large and cannot be directly assessed in direct numerical simulations (DNS) but require an asymptotically reduced equation valid in conditions of sufficiently fast background rotation (Sprague et al. 2006; Julien et al. 2012). The Rayleigh number, defined as the ratio of the thermal buoyancy to the viscous force, can be expressed in the form

$$
R a=\frac{g \beta \Delta T H^{3}}{\kappa \nu},
$$

where $g, \Delta T$, and $H$ are the acceleration of gravity, the temperature difference between the top and bottom boundaries, and the height of the fluid layer; $\beta, \kappa$ and $\nu$ represent the thermal expansion coefficient, the thermal diffusivity, and the kinematic viscosity. The effect contributed by rotation is frequently dictated by the Taylor number Ta, defined as the ratio of the Coriolis force to the viscous force and expressed by

$$
T a=\left(\frac{2 \omega H^{2}}{\nu}\right)^{2},
$$

where $\omega$ is the angular velocity of the background rotation. Flow structures undergoing rotating $\mathrm{RBC}$ are commonly categorized with the convective Rossby number Ro, representing the contribution of the rotation relative to the thermal force,

$$
R o=\frac{1}{2 \omega} \sqrt{\frac{g \beta \Delta T}{H}}=\sqrt{\frac{R a}{\operatorname{Pr} T a}} .
$$

To overcome the constraints imposed by computational limitations stemming from the widely distributed values of geophysical or astrophysical parameters, Sprague et al. (2006) proposed an asymptotic reduction for a rapidly rotating RBC. In this manner, smaller Ro conditions including actual geophysical or astrophysical states can be determined. For instance, Aurnou et al. (2015) predicted heat transport under conditions similar to interior Earth conditions by extrapolating the Nusselt and Rayleigh number $(N u-R a)$ relation. The flow structures in rapidly rotating $\mathrm{RBC}(R o \ll 1)$ have been investigated (Grooms et al. 2010; Stellmach et al. 2014; Cheng et al. 2015; Julien et al. 2016). Recent laboratory experiments have attempted to reach $R a$ and $T a$ regimes where asymptotic theory can be applied (Cheng et al. 2018). Here, the flow states in rapidly rotating RBC with Prandtl numbers, $\operatorname{Pr}=\nu / \kappa>0.2$, may be categorized into four regimes. With decreasing $R o$ values, they are three-dimensional (3D) turbulence, vortical plumes, convective Taylor columns (CTC), and cellular regimes identified by the variations in $N u$ (Liu \& Ecke 2009; Weiss \& Ahlers 2011; Julien et al. 2012; Stevens et al. 2013). In vortical plumes and CTC regimes, the columnar vortex structures dominate the flow fields. Julien et al. (2012) established that steady columnar vortices do not emerge for $\operatorname{Pr} \leqslant 3$. Prior to relatively recent works mentioned elsewhere, earlier reports had established the flow structures associated with transient regimes where the range of $R a$ is sufficiently large to induce finite-amplitude convection and also sufficiently small to yield prominent geostrophic flows. Under these conditions, up-welling and down-welling columnar vortex behaviour is a result of the interplay between the surrounding columns (vortical plumes). Sakai (1997) reported on theoretical models describing the horizontal scale of columnar vortices at $R o \sim 10^{0}-10^{-1}$. The motion of the columnar vortices was also recorded in the 
Sakai (1997) experimental reports. Further details of the vortex motion were reported in earlier work by Zhong et al. (1993). Here the mutual interaction (merging phenomena) of vortical structures in advection as time variations of the distance and angular orientation between two adjacent vortices were investigated, calculated from multiple vortex positions using flake visualization. Julien et al. (1996) presented a breakdown of the cellular roll state for $R o=0.75$ using DNS, and observed intense chaotic mutual interactions between vortices leading to merging and vanishing. Such mutual interactions were explained as the result of lateral mixing, which maintains an unstable horizontal mean temperature gradient in the fluid layer. Vorobieff \& Ecke $(1998,2002)$ visualized spin-up states of rotating $\mathrm{RBC}$ and found advective motion of the vortical structures in the $0.2<R o<1$ regime. Despite the many reports on such vortex motion, a detailed statistical analysis of the motion of columnar vortices has remained little understood.

In investigating vortex motion in a laboratory setting, the effect of centrifugal forces must be considered (Cheng et al. 2018). Conventionally, the Froude number is used to describe the effect of centrifugal forces. Defined as the ratio of the centrifugal force to the gravitational force,

$$
F r=\frac{L \omega^{2}}{2 g},
$$

with $L$ is the diameter of the cylindrical fluid layers, this factor cannot be disregarded in laboratory experiments because here the finite size of the fluid layers strongly influence the fluid motion. Horn \& Aurnou (2018) analysed a wide range of DNS of Corioliscentrifugal convection $\left(\mathrm{C}^{3}\right)$, and discussed centrifugal forces by defining a centrifugal Rossby number, $R o_{c}=\operatorname{Ro} \sqrt{2 \mathrm{Fr} / \Gamma}$, where $\Gamma$ denotes the aspect ratio of the fluid layer given as $\Gamma=L / H$. In such arrangements, centrifugally dominated convection occurs for $\mathrm{Fr}>\Gamma / 2$. To be able to disregard centrifugal effects, the aspect ratio of the fluid vessel must be small in rapidly rotating RBC (Cheng et al. 2018). For empirical criteria, a condition with $\mathrm{Fr}<0.1$ is thought to allow disregarding the contribution of centrifugal force in rotating $\mathrm{RBC}$, however, this has not been finally verified. One reason is the long timescales of the effects of centrifugal buoyancy $O\left(10^{2}-10^{3} \mathrm{~s}\right)$ in laboratory settings (Horn \& Aurnou 2018). Noto et al. (2018) established a vortex detection method employing visualization of instantaneous temperature fields enabling the prolonged tracking of columnar vortices. In conventional vortex identification, values for the velocity fields must be established because the vortex structures may be determined by the vorticity fields or other quantities such as the $Q$ criteria (Kunnen et al. 2010; Stevens et al. 2012; Kunnen et al. 2014). Particle image velocimetry (PIV) is a commonly applied method to measure two-dimensional (2D) velocity fields. This method measures the instantaneous velocity vector field by identifying local similarities between two sequential images captured within a short time interval. Here the time interval required depends on the flow velocity, and typically rates of $10-100$ frame per second (f.p.s.) are used for thermal convection measurements. Without considering appropriate time intervals to track vortices, data storage and/or computational load would be potential difficulties. In addition, the spatial resolution of the velocity (vorticity or $Q$-value) fields become less detailed than the original digital images. Thus, a PIV approach does not necessarily suit to measurements of vortex motion conducted over extended time periods: from such considerations the method proposed by Noto et al. (2018) becomes an advantageous.

The objective of this study is to establish the translational motion of the quasi-2D columnar vortices formed in rotating RBC with and without centrifugal force effects based on the measurement technique established in Noto et al. (2018). The flow regimes at $R o \sim 10^{0}-10^{-1}$ are chosen to display the active motion of the columns, as reported 
(a)

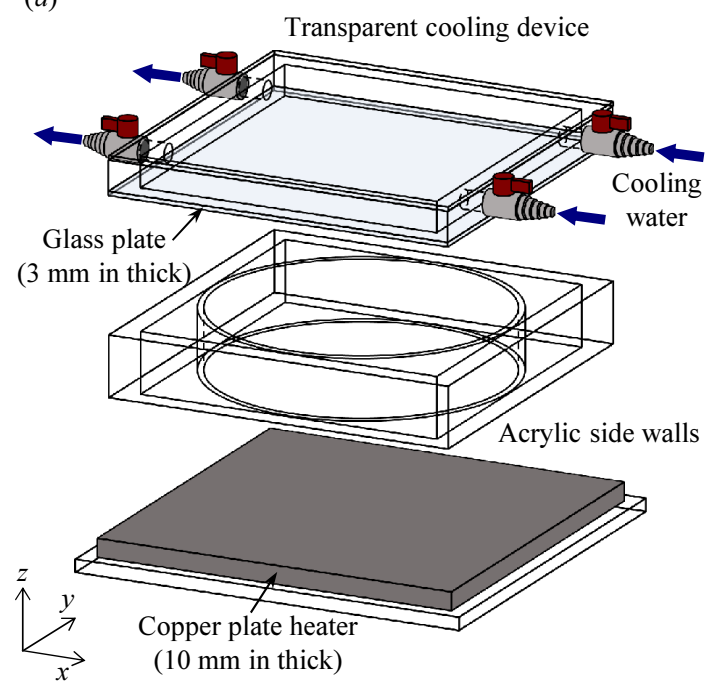

(b) Unit: $\mathrm{mm}$

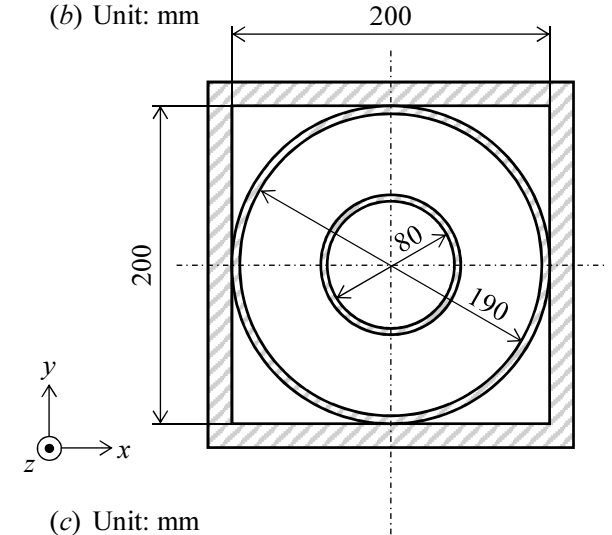

(c) Unit: $\mathrm{mm}$

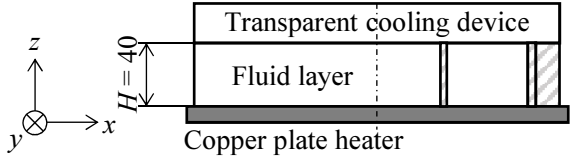

FIGURE 1. Schematic diagram of the fluid vessel: $(a)$ Overview of the vessel with the acrylic walls, cooling device, and copper plate heater. The cylinder inside the square walled vessel is that of $L=190 \mathrm{~mm}$. (b) Horizontal cross-section of the vessel, where two different cylinders ( $L=80$ or $190 \mathrm{~mm}$ ) are alternatively placed. (c) Vertical cross-section of the vessel.

previously (Zhong et al. 1993; Julien et al. 1996; Sakai 1997). In these regimes, it has not been established whether the conventional criteria identifying the four different flow regimes applies to laboratory experiments. To ensure the generation of columnar vortices in the parameter ranges investigated, the flow structures in the vertical cross-sections were initially investigated by measuring the velocity fields. Under conditions where columnar vortical structures develop, the horizontal motion of these structures, labelled by the method proposed in Noto et al. (2018), were tracked for longer than $10^{3} \mathrm{~s}$. Finally, a detailed discussion on the horizontal translational motion of the columnar vortices is presented based on the results of the statistical analyses here.

\section{Experimental setup and measurement method}

\subsection{Experimental setup}

The fluid vessel used in the present study is shown in figure 1 . The side walls enclose an acrylic square vessel $200 \mathrm{~mm}$ wide and $40 \mathrm{~mm}$ high. A cylindrical fluid layer of the same height, $H=40 \mathrm{~mm}$, was formed by placing one of two acrylic cylinders (diameters $L=80$ and $190 \mathrm{~mm}$ ) in the square vessel allowing easy change in the horizontal geometries. Degassed distilled water $\left(\operatorname{Pr} \sim 7\right.$ at $\left.20^{\circ} \mathrm{C}\right)$ was used as the test fluid. A $10 \mathrm{~mm}$ thick copper plate heater was set under the bottom of the vessel. Isothermal heating was maintained by controlling the electric current supplied to the heater. The top surface of the vessel was cooled by circulating water from a thermostatic bath via a $3 \mathrm{~mm}$ thick glass plate. The corresponding Biot number in the present study is around 0.6 (Noto et al. 2018), and preliminary experiments verified the homogeneity of the top cooling condition with this glass plate thickness. The effective temperature difference across the fluid layer was $\Delta T=11.1^{\circ} \mathrm{C}$ measured by thermistors located at the bottom of the copper plate and right beneath the top glass plate. The fluid vessel and all the optical equipment including a camera were mounted on a rotating platform (1-m diameter with 
(a)
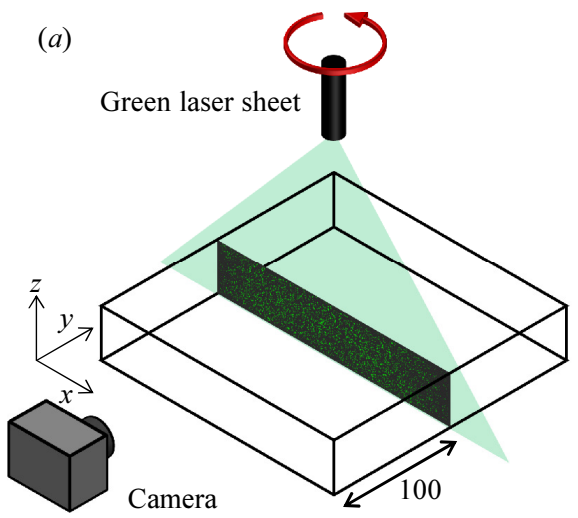

(b)

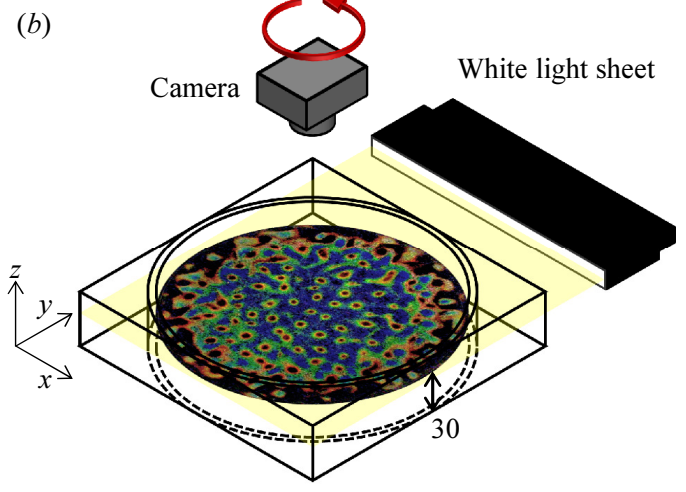

FIgURE 2. Optical arrangements for $(a)$ the vertical velocity field measurements by PIV and (b) the horizontal temperature field measurements by TLC particles. Images embedded in each panel are examples obtained by these two different visualization methods. For the experiments the fluid vessel and optical equipment was placed on a platform and rotated as suggested by the red arrows.

two stages, not shown in figure 1) and rotated together with the vessel and plates. A detailed description of the present experimental apparatus for this rotating RBC was given elsewhere (Noto et al. 2018). The angular velocity $\omega$ realized by this setup is from $0.0-5.0 \mathrm{rad} / \mathrm{s}$, corresponding to $T a=0.0-2.8 \times 10^{8}$. Using different configurations of the optical equipment as suggested in figure 2, two different series of experiments were conducted: velocity field measurements of a vertical cross-section (figure $2 a$ ) and temperature field measurements of a horizontal cross-section (figure $2 b$ ). Details of the experiments are described separately in the following sections.

\subsection{Velocity field measurements on a vertical cross-section}

To perform the PIV measurements of the velocity field on a vertical cross-section, a 2 -W green laser sheet $(<5$-mm thick) is illuminated from above the cross-section at the middle position $(y=2.5 H, 100 \mathrm{~mm}$ ) of the fluid layer (figure $2 a$ ). Without any of the cylinders was placed in the acrylic vessel during the PIV measurements. A small amount of porous resin particles (mean diameter $d_{p}=90 \mu \mathrm{m}$, mean density $\rho_{p}=1.02 \times 10^{3} \mathrm{~kg} / \mathrm{m}^{3}$, HP20SS, Mitsubishi Chemical Co.) were dispersed into the test water and used as flow tracers. The Stokes number $S t=\rho_{p} d^{2} U /(18 \rho \nu H)$ is $O\left(10^{-4}\right) \ll 1$, with $\rho$ the density of the working fluid and $U$ the representative flow velocity (to be detailed below); this will ensure that the tracer particles follow the fluid motions closely. From one side of the vessel, particle images in the $x-z$ plane were recorded using a digital camera (EXF1, CASIO) at 5 f.p.s. and a resolution of $0.1 \mathrm{~mm} /$ pixel $(2000 \times 400$ pixels for $200 \times 40$ $\mathrm{mm}^{2}$ ). The images are processed using a standard cross-correlation method to derive instantaneous velocity vector fields from two sequential images.

\subsection{Temperature field measurements on a horizontal cross-section}

For the temperature field visualization, a white light sheet generated by a halogen lamp illuminated a horizontal cross section at a set height, $z=0.75 H(30 \mathrm{~mm})$, of the fluid layer (figure 2b). A camera, the same as the one used for the PIV experiments, was set at the axis of rotation and recorded colour images of the $x-y$ plane from above the fluid layer. Encapsulated thermochromic liquid crystals (TLC) particles (mean diameter $d_{p}=15 \mu \mathrm{m}$, mean density $\rho_{p}=1.02 \times 10^{3} \mathrm{~kg} / \mathrm{m}^{3}$, the approximate dynamic 


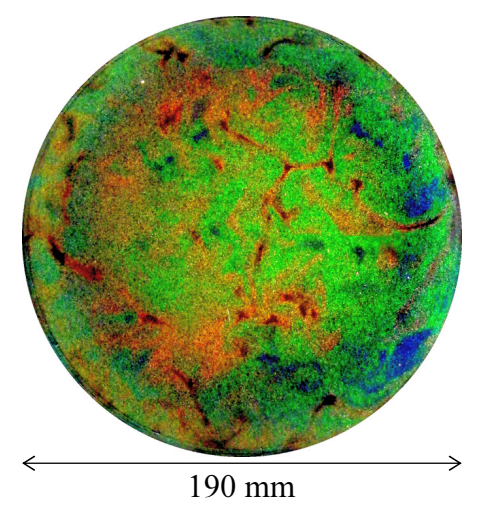

Figure 3. An example of a horizontal temperature field visualized using the encapsulated TLC at $z=0.75 H$ for $R a=1.0 \times 10^{7}$, and $T a=0$ recorded in the $\Gamma=4.75$ vessel.

range in $20-25^{\circ} \mathrm{C}$, Japan capsular products inc.) were suspended in degassed water for the temperature field visualization. Estimated $S t, O\left(10^{-6}\right)$, ensures sufficiently high traceability of the TLC particles. An example of a temperature field visualized by TLC at no-rotation conditions $\left(R a=1.0 \times 10^{7}\right.$ and $\left.T a=0\right)$ obtained in the larger vessel is shown in figure 3. The TLC shows colouration corresponding to the temperature by the scattered incident white light. Typically, red is at the lower end of the colouring temperature range, and the TLC changes scattering colour, from green, light blue, to dark blue as the temperature increases. As shown in figure 3 , it is possible to observe red-coloured, cold down-welling flows, and blue-coloured, warm up-welling flows showing the large-scale structures in this condition. The working fluid filled the entire fluid layer including the corner regions outside the cylinders. As a result, the temperature of the ambient fluid is controlled within the set temperature differences playing the role of a water jacket, as well as this reduces unwanted reflection of the incident light and provides better quality colour images. The colour images were acquired at 3 second intervals $(\Delta t=3 \mathrm{~s}, 1 / 3$ f.p.s.) with the f-number 2.7 , and a resolution of $0.1 \mathrm{~mm} /$ pixel for $\Gamma=2.00$ and $0.1357 \mathrm{~mm} /$ pix for $\Gamma=4.75$. The TLC is useful to observe the fluid temperature noninvasively, its calibration from the colour information to temperature is influenced by a number of uncertainties (Wiberg \& Lior 2004; Rao \& Zang 2010; Abdullah et al. 2010). For instance, sensitivity to the incident light yields colourations with a strong dependence on image positions, and the observation direction (the angle measured from the lens) induces similar colour inhomogeneities. To overcome these TLC measurement difficulties, the present study employed localized hue calibration to obtain the temperature fields and applied a robust vortex detection method (Noto et al. 2018). In this, the temperature at each pixel was calibrated from locally obtained hue-temperature calibration curves. By combining the vortex detection method with this calibration, it is possible to identify columnar vortices from horizontal temperature fields as undulations at $\operatorname{Pr} \sim O(1)$.

\section{Development of quasi-2D columnar vortices}

\subsection{Vertical coherence of vortical structures}

Columnar vortical structures, the main focus of the present study, are observed in the transition from a 3D thermal turbulence regime to a CTC regime via a vortical plume regime (Grooms et al. 2010). The emergence of the columnar vortices requires (quasi- 


\begin{tabular}{|c|c|c|c|c|c|c|}
\hline \multirow{2}{*}{$\operatorname{Pr}$} & \multirow{2}{*}{$R a$} & \multirow{2}{*}{$T a$} & \multirow{2}{*}{ Ro } & \multirow{2}{*}{$F r$} & \multicolumn{2}{|c|}{$N_{\text {vortex }}$} \\
\hline & & & & & Up-welling & Down-welling \\
\hline \multirow{5}{*}{$\sim 7\left(\right.$ at $\left.20^{\circ} \mathrm{C}\right)$} & \multirow{5}{*}{$1.0 \times 10^{7}$} & $1.0 \times 10^{6}$ & 1.20 & $1.0 \times 10^{-3}$ & 16.0 & 52.6 \\
\hline & & $6.0 \times 10^{6}$ & 0.49 & $6.0 \times 10^{-3}$ & - & - \\
\hline & & $1.0 \times 10^{7}$ & 0.38 & $1.0 \times 10^{-2}$ & 74.7 & 114.2 \\
\hline & & $6.0 \times 10^{7}$ & 0.15 & $6.0 \times 10^{-2}$ & - & - \\
\hline & & $1.0 \times 10^{8}$ & 0.12 & $1.0 \times 10^{-1}$ & 198.8 & 214.2 \\
\hline
\end{tabular}

TABLE 1. Parameter settings for the vertical velocity measurements, and measurements of the number of up-welling and down-welling vortices, from Noto et al. (2018).

geostrophic conditions that are conventionally assumed at $R o \ll 1$. But the parameter boundaries between the regimes must be evaluated in detail in the experiments, and are considerably influenced by side boundaries and centrifugal forces. Consequently, five different conditions, as detailed in table 1, were investigated to evaluate details of the emergence of columnar vortices by PIV measurements. Due to the limitations of the turn table, the Ro distributes from 1.20 to 0.12 for the set Rayleigh number, $R a=1.0 \times 10^{7}$, in the test conditions here. In the following, $N_{\text {vortex }}$ is the number of columnar vortices determined in the temperature-field image at the horizontal cross-sections of $z=0.75 \mathrm{H}$ inside a cylinder with $\Gamma=4.75$ (Noto et al. 2018). The number of heated vortices present under very similar conditions, $R o=0.1-10$, in the DNS conducted by Pieri et al. (2016), is similar to that obtained here (see table 1 ). Typically, $N_{\text {vortex }}$ increases with decreasing $R o$, and $N_{\text {vortex }}$ strongly depends on the vertical height position. To quantify the vertically distributed characteristics, Rajaei et al. (2017) reported that an increase in the vertical coherence with decrease in $R o$ by PIV experiments. This suggests that the development of columnar vortices may be observed in decreasing $R o$ conditions in the images of the vertical velocity fields.

Examples of the velocity vector distributions measured in the $x-z$ plane at $y=2.5 H$ for each $T a$ setting are shown in figure 4. Here, the magnitude of the measured velocity $\sqrt{u^{2}+w^{2}}$ is rendered in grey scale. Flow structures at low Ta settings, as in figure $4 a$, $b$, and $c$ are not well organized along the direction of the rotational axis, and only some column-like structures are visible. At high $T a$ settings, figure $4 d$ and $e$, flow structures are recognizable as separate columns. A representative flow velocity is given by Prandtl's free-fall theory, $U_{f}=\sqrt{g \beta \Delta T H}$, and here estimated at roughly $U_{f} \sim 30 \mathrm{~mm} / \mathrm{s}$. The magnitude of the velocity in rotating $\mathrm{RBC}$ is small, at almost only one tenth of $U_{f}$ because the background rotation is suppressed even when $T a$ is the smallest. This is also indicated from the fact that the magnitude of the velocity for higher $T a$ values is lower than that of the lower $T a$. A typical geostrophic velocity proposed by Sakai (1997), $U_{0}$, reflecting a geostrophic balance in the fluid interior, may be obtained from

$$
U_{0}=\frac{\kappa}{2 l}\left(\frac{\Delta T_{c}}{\Delta T}\right) R a T a^{-1 / 2},
$$

where $\Delta T_{c}$ is the temperature difference between up-welling and down-welling vortices when the vertical temperature of the columns is constant, and the columns are separated by a distance $l$. Under the present conditions, $U_{0}$ is of the order $O(1 \mathrm{~mm} / \mathrm{s})$, which would agree well with the present results.

To highlight the vertical coherence of the flow structures, the corresponding distributions of the vertical velocity $w$ for the images shown in figure 4 were also calculated and 


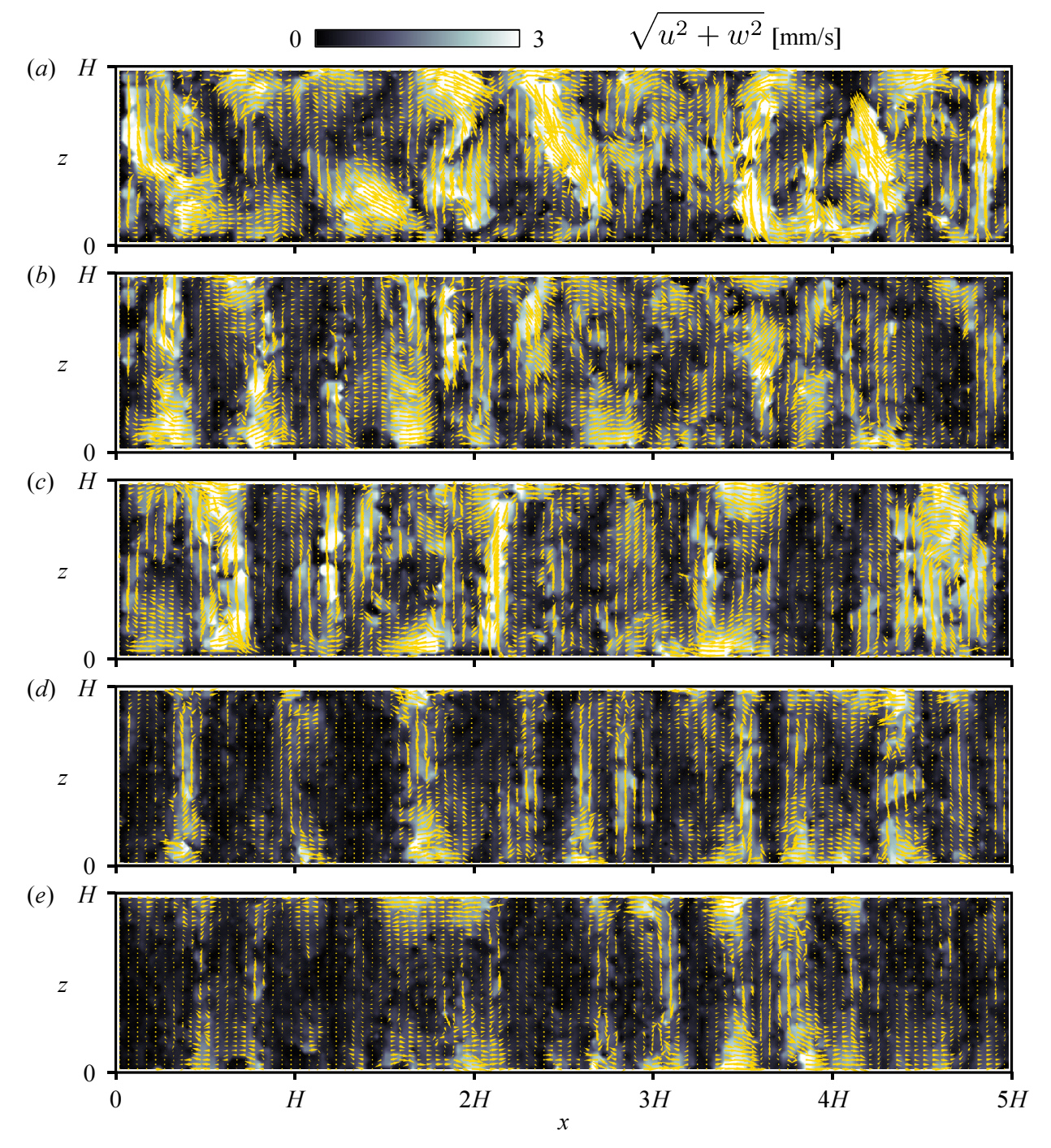

Figure 4. Snapshots of the velocity vector distributions of vertical cross-sections measured in PIV at $y=2.5 H:(a) T a=1.0 \times 10^{6}(R o=1.20),(b) T a=6.0 \times 10^{6}(0.49),(c) 1.0 \times 10^{7}(0.38)$, (d) $6.0 \times 10^{7}(0.15)$, and $(e) 1.0 \times 10^{8}(0.12)$. Only every second vector (the slanted yellow line) is plotted for clearer visibility.

are shown in figure 5. While somewhat disorganized, structures are visible in figure $5 a$, these are vertical coherent structures from bottom to top (bright), and from top to bottom (dark), aligned alternately in horizontal directions in the other cases satisfying $R o<1$. These indicate up-welling and down-welling flows that arise from Ekman pumping. The structures appear increasingly regularized in the vertical direction with increasing $T a$; the horizontal distance (left-right in the figure) between the structures also becomes shorter. In the previous report (Noto et al. 2018), the distance between neighbouring structures at each setting was found to agree well with the theory established by Sakai (1997). Fluctuations of the velocity in the vertical direction diminish with $T a$ with the structure appearing as a quasi-2D regular column in figures $5 d$ and $e$, specifically, at $R o=0.15$ 
$-3 \square 3 \quad w[\mathrm{~mm} / \mathrm{s}]$

(a)

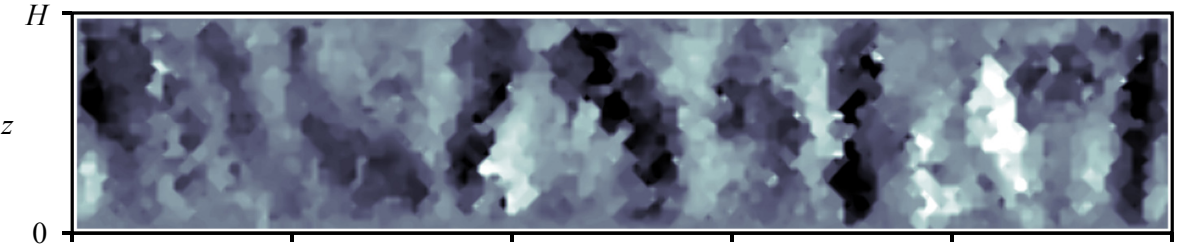

(b)

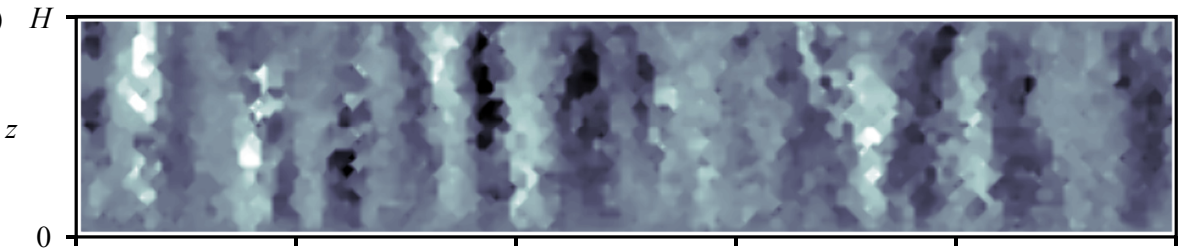

(c)

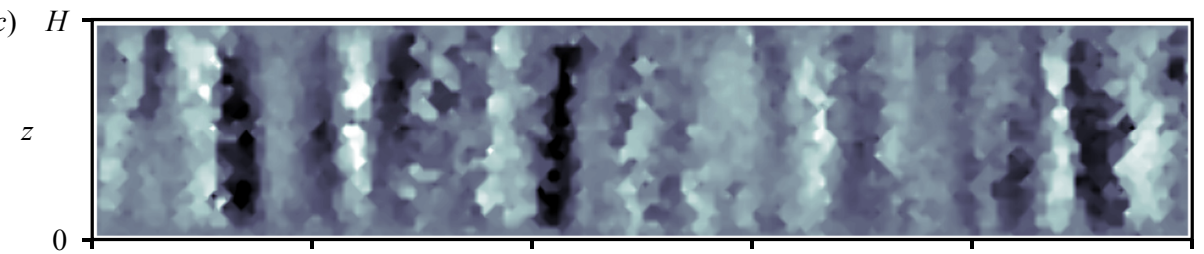

(d)

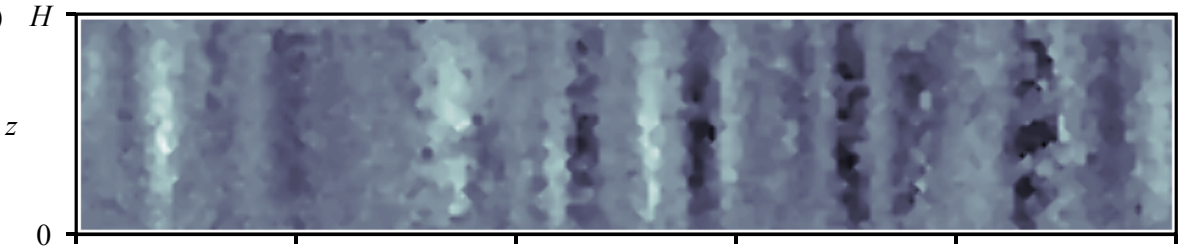

(e)

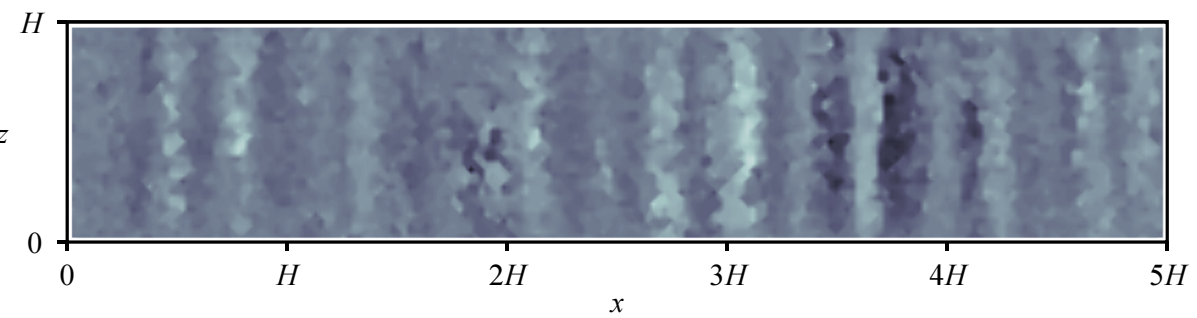

Figure 5 . Vertical velocity distributions $w(x, z)$ in vertical cross-sections at the same moment to that of figure 4 measured by PIV at $y=2.5 H:(a) T a=1.0 \times 10^{6}(R o=1.20)$, (b) $T a=6.0 \times 10^{6}(0.49),(c) 1.0 \times 10^{7}(0.38),(d) 6.0 \times 10^{7}(0.15)$, and $(e) 1.0 \times 10^{8}(0.12)$.

and 0.12. Further quantitative criteria to distinguish prominent columnar vortices are discussed in the next section.

\subsection{Local duration characteristics of columnar vortices}

In the previous section, spatial, instantaneous quasi-2D columnar structures were analysed. To investigate the translational motion of columnar vortices, the duration of vertical coherence also needs to be investigated in regard to the time variation in the appearance. This is required to verify that the vortices detected on the horizontal plane in sequential images exhibit translational motion and not a continuous skewed deformation. This also has importance in determining a suitable time resolution for vortex tracking. One advantage of TLC visualization is that detection of the vortices may be achieved from a single instantaneous image with a wider flexible range of time resolutions than 


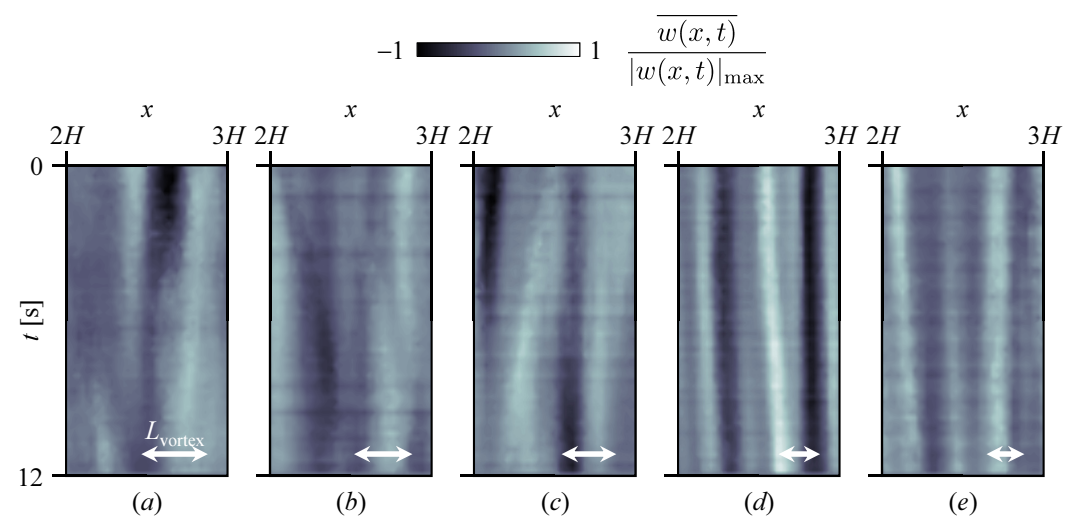

FiguRE 6 . Time series of averaged vertical velocities at horizontal positions $2 H<x<3 H$ : (a) $T a=1.0 \times 10^{6}(R o=1.20),(b) T a=6.0 \times 10^{6}(0.49),(c) 1.0 \times 10^{7}(0.38),(d) 6.0 \times 10^{7}(0.15)$, and $(e) 1.0 \times 10^{8}(0.12)$. Two-headed arrows indicate the theoretically predicted diameter of the columnar vortices $L_{\text {vortex }}$ derived by Sakai (1997).

in PIV requiring two consecutive images taken within a short time interval (Noto et al. 2018).

Considering the results obtained with PIV, in particular those in figures $5 d$ and $e$, the sign of the vertical velocity $w$ along a single columnar vortex is the same from top to the bottom and in the image. This is because all the columnar vortices can be classified as up-welling $(w>0)$ or down-welling $(w<0)$ vortices. As Rajaei et al. (2017) showed, vertical coherence of columnar vortices can be obtained by making autocorrelations of vorticity along the rotational axis, when all of the velocity information (three-dimension and three-component) is available. In the present study, the available information are the velocity field only in the $x-z$ plane, and it is necessary to show vertical coherence of the columnar vortices in this condition. Hence, the averaged vertical velocity $\overline{w(x, t)}=\frac{1}{H} \int_{0}^{H} w(x, z, t) d z$ is a useful indicator to identify column formation. To establish the time variation of the vertical coherence, the evolution of $\overline{w(x, t)}$ with elapsed time is plotted (figure 6). In figure 6 the values have been normalized using the maximum of the absolute value. The two-headed arrows in each panel are equivalent to the theoretically predicted diameters of the vortices, $L_{\text {vortex }}$, derived by Sakai (1997) and as will be defined below. In figures $6 a$ and $b$, the bright and dark stripes do not maintain the same width throughout the observation period. A zero value of $\overline{w(x, t)}$ indicates the absence of columns. With a larger $T a$ setting, figures $6 c, d$, and $e$, show a straight striped pattern. The columns appear at very similar positions, at least within the observation period for the larger $T a$ settings here. In addition, these are variations in the width of the stripes. Assuming that the widths of the columnar vortices are constant in the horizontal direction, these variations arise from plane displacements, specifically, column displacements in the $y$-direction; skewed stripes indicate displacements in the $x$-direction.

Assuming that the horizontal motion of the vortices is isotropic, the advection speed of the columnar vortices may be estimated simply from the slopes of the stripes,

$$
v_{\mathrm{adv}}=\sqrt{\left(\frac{d x}{d t}\right)^{2}+\left(\frac{d y}{d t}\right)^{2}} \sim \sqrt{2\left(\frac{d x}{d t}\right)^{2}},
$$

with $v_{\text {adv }}$ indicating the horizontal advection speed of the columnar vortices. From these 


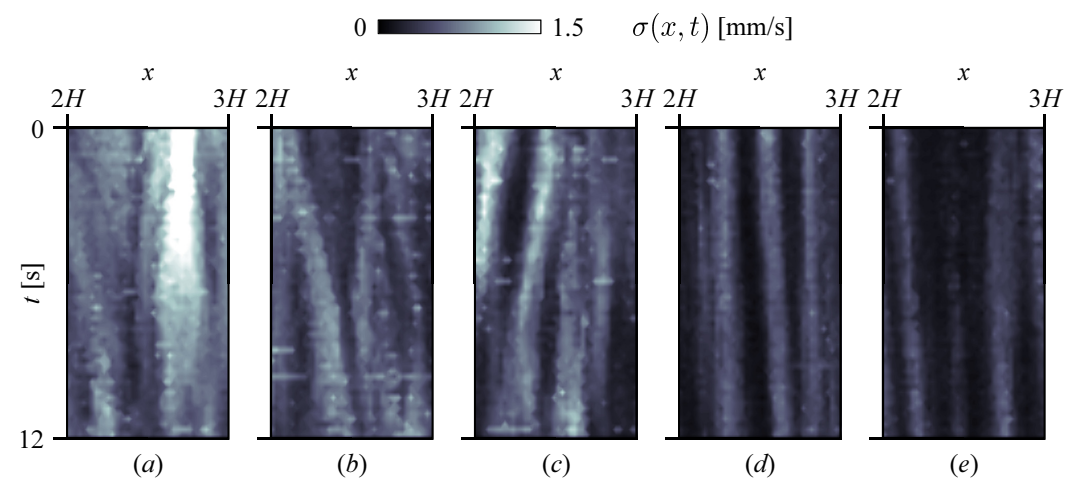

Figure 7. Time series of the standard deviations for the vertical velocity component at horizontal positions $2 H<x<3 H:(a) T a=1.0 \times 10^{6}(R o=1.20),(b) T a=6.0 \times 10^{6}$ $(0.49),(c) 1.0 \times 10^{7}(0.38),(d) 6.0 \times 10^{7}(0.15)$, and $(e) 1.0 \times 10^{8}(0.12)$.

estimates of the maximum value of $v_{\text {adv }}$ is $O(0.1-1 \mathrm{~mm} / \mathrm{s})$. With this, the time resolution for the vortex tracking of the horizontal cross-sections becomes $O(1 \mathrm{~s})$ under the present optical conditions.

In addition to the vertical average, the standard deviation of $w$, obtained from $\sigma(x, t)^{2}=\frac{1}{H} \int_{0}^{H}[w(x, z, t)-\overline{w(x, t)}]^{2} d z$, gives another estimate of the vertical coherence, the standard deviation, $\sigma(x, t)$, for each of the periods in the images of figure 6 are presented in figure 7. At lower Ta settings, the high magnitude of $\sigma(x, t)$ indicates that $w$ varies along the z-axis. Accordingly, if the vertically straight (coherent) columns maintain their structures during this observation time, the magnitude of $\sigma(x, t)$ does not change at the same horizontal positions. For this reason, it is difficult to identify column possessing system height structures for the lowest two Ta settings. A spatiotemporal integration (average) of $\sigma(x, t), \bar{\sigma}=\frac{1}{L T} \int_{0}^{L} \int_{0}^{T} \sigma(x, t) d x d t$, suggests how stable the columnar vortices are allowing the local characteristics of the duration of the columnar vortices to be quantified. This is because a smaller value of the spatiotemporal integration of the vertical variance signifies vertical coherence of the columnar vortices. The variations in $\bar{\sigma}$ with $T a$ are shown in figure 8; here, the dashed line shows the fitted curve calculated by the least-squares method. In the present study, the curve obtained is $\bar{\sigma} / U_{f}=0.026 R o^{0.47}$. As $R o$ approaches zero, the flow structures maintain vertical coherence here. For low Ro values there is a cellular regime (Cheng et al. 2015), where no visible vortex motion appears. This suggests that the results obtained in this analysis may be considered reasonably accurate. In figure 8 , the ratio of $\bar{\sigma}$ to $U_{f}$ is $\sim 0.01$ for the two highest $T a$ settings, with only $1 \%$ of deviation from the free-fall velocity $U_{f}$.

To track the translational motion of the columnar vortices further, the points discussed in this section may be summarized as follows. The vortical structures exhibit two dimensionality in the velocity distributions on the vertical cross-sections. Skewed structures along the $z$-axis represent a $3 \mathrm{D}$ turbulence-like state for the lowest $T a$ settings. With the time variation of $w$, a horizontal advection speed of the columns, $v_{\text {adv }}$, could be estimated. The estimated value of $O(0.1-1 \mathrm{~mm} / \mathrm{s})$ suggests an appropriate time resolution for vortex tracking of every few seconds ensuring that the detailed time resolution avoids erroneous tracking. In addition, the number of up-welling and down-welling vortices is also a good indicator of the flow state. An almost equal number of these in the same horizontal crosssection indicates (fully) developed CTC (see table 1). In summary, columnar vortices with 


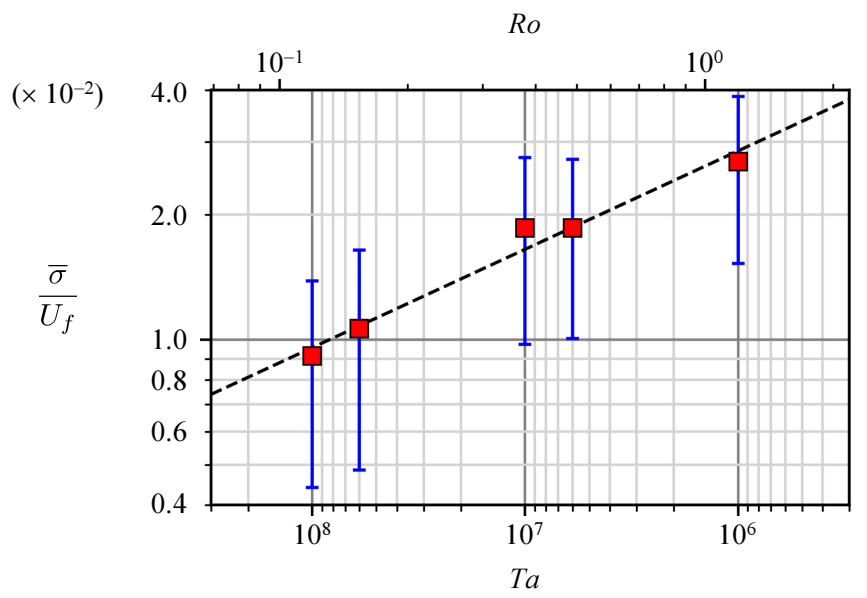

FiguRE 8. Variation of local duration characteristics $\bar{\sigma}$ plotted with error bars giving the standard deviation. Here, $\bar{\sigma}$ is normalized by the free-fall velocity $U_{f}$. The dashed line is a least-squares fit of $\bar{\sigma} / U_{f}=0.026 R o^{0.47}$

\begin{tabular}{cccccc}
$\operatorname{Pr}$ & $R a$ & $\Gamma$ & $T a$ & $R o$ & $F r$ \\
\hline \hline & & & $1.0 \times 10^{7}$ & 0.38 & $4.0 \times 10^{-3}$ \\
& & 2.00 & $3.0 \times 10^{7}$ & 0.22 & $1.2 \times 10^{-2}$ \\
$\sim 7$ & $1.0 \times 10^{7}$ & & $1.0 \times 10^{8}$ & 0.12 & $4.0 \times 10^{-2}$ \\
\cline { 3 - 6 } & & $1.0 \times 10^{7}$ & 0.38 & $9.5 \times 10^{-3}$ \\
& & 4.75 & $3.0 \times 10^{7}$ & 0.22 & $2.9 \times 10^{-2}$ \\
& & & $1.0 \times 10^{8}$ & 0.12 & $9.5 \times 10^{-2}$
\end{tabular}

TABle 2. Parameter settings for the vortex tracking experiments.

$T a \geqslant 1.0 \times 10^{7}(R o \leqslant 0.15)$ ensure a horizontal translational motion of the columnar vortices in the present study.

\section{Translational motion of columnar vortices}

Next, we investigated the horizontal translational motion of the quasi-2D columnar vortices. Unlike the PIV experiments (section 3), circular fluid layers of different radii, $D=80$, and $190 \mathrm{~mm}$, with the same height (thickness), $H=40 \mathrm{~mm}$ were selected for examining the variation in the Froude number $F r$ for different values of the aspect ratio $\Gamma$ given the same rotational parameter settings. The experimental settings for the vortex tracking are listed in table 2 . For set $R a$ values, $R a=1.0 \times 10^{7}$, three different $T a$ values in the range of $1.0 \times 10^{7} \leqslant T a \leqslant 1.0 \times 10^{8}$ were set. The corresponding values for $\mathrm{Fr}$ are in the range of $4.0 \times 10^{-3} \leqslant F r \leqslant 9.5 \times 10^{-2}$. The effect of the centrifugal force has been empirically disregarded in cases of $F r<0.1$ (Cheng et al. 2018), and even the largest $F r=9.5 \times 10^{-2}$ in the present study is smaller than 0.1 . This suggests that the effect of the centrifugal force may be considered negligible for all the conditions here according to empirical criteria. 


\subsection{Vortex detection from horizontal temperature field}

Using encapsulated TLC particles, we were able to develop images of the temperature field (figure 9) to visualize the columnar vortices. Heated up-welling and cooled downwelling vortical structures are simply recognized as blue and red circular colour variations. For both $\Gamma$ instances, finer and more-organized structures with increasing $T a$ were found to be common changes. The horizontal scale of these vortical structures, denoting $L_{\text {vortex }}$, was predicted in previous work. At the onset of convection, Chandrasekhar (1961) using linear stability theory derived a $L_{\text {vortex }}=c H T a^{-1 / 6}$ with the prefactor $c=4.8$. Differently, Cheng et al. (2018) found $c=2.4$ taking Ekman pumping into account with $T a<10^{14}$. In water experiments without a rigid top boundary, Boubnov \& Golitsyn (1986) found an empirical scaling $L_{\text {vortex }}=R a^{1 / 9} \mathrm{Ta}^{-1 / 4}$ using the present notation. Sakai (1997) derived a theoretical scaling law by considering a physical model with two rigid boundaries and verified its adequacy experimentally. From his estimate, the horizontal scale of columnar vortices $L_{\text {vortex }}$ is

$$
L_{\text {vortex }}=4.6 H \frac{\delta_{t}^{2}}{2 \delta_{t}^{2}+2 \delta_{t} \delta_{E}+\delta_{E}^{2}} R a^{1 / 3} \mathrm{Ta}^{-3 / 8},
$$

where $\delta_{t}$ and $\delta_{E}$ denote the thickness of the thermal and Ekman boundary layers, respectively. Under set $R a$ conditions, $L_{\text {vortex }}$ depends on only $T a$. Our previous work (Noto et al. 2018) established a vortex determination method from instantaneous temperature fields using this estimate. On actual colour images, temperature values are reliably related to hue values, which directly shows the colour itself as a degree like $0^{\circ}$ for red, $120^{\circ}$ for green and $240^{\circ}$ for blue. This is useful to calibrate colouration to temperature, even though the uncertainty is not negligible. In the horizontal temperature fields obtained through this colour-temperature calibration, columnar vortices can be regarded as temperature undulations similar to a point spread function - a 2D Gaussian distribution. This is because the temperature inside the down-welling (or up-welling) vortex gradually decreases (increases) toward the centre, and these facts result in the concave (convex) temperature distribution inside the vortex (e.g., figure 6 in Noto et al. 2018). Such concave or convex undulations can be extracted using pattern recognition techniques. In the present study, the same method was employed to detect columnar vortices of horizontal cross-sections.

\subsection{Statistical characteristics of advection velocity of columnar vortices}

As $R o$ approaches zero, the number of up-welling and down-welling vortices approaches a balance (Vorobieff \& Ecke 2002). Assuming a balanced population and columnar vortices in vertical coherence, then only one, for example, the down-welling vortex motion needs to be investigated (The centrifugal force discussed below, however, may affect statistical motions of up- and down-welling vortices and push vortices in the opposite directions.). Image acquisition was conducted for a one-hour period after reaching a fully developed flow state (more than 30 minutes from initiating the rotation). Around 1200 consecutive images and temperature fields were obtained. The nearest-neighbour method was employed to track the labelled vortices on the visualized temperature fields, in which two vortices having the shortest Euclidean distance between two consecutive images are linked. The vortex advection speed $v_{\text {adv }}$ can then be obtained using

$$
v_{\mathrm{adv}}=\frac{|\boldsymbol{r}(t+\Delta t)-\boldsymbol{r}(t)|}{\Delta t},
$$



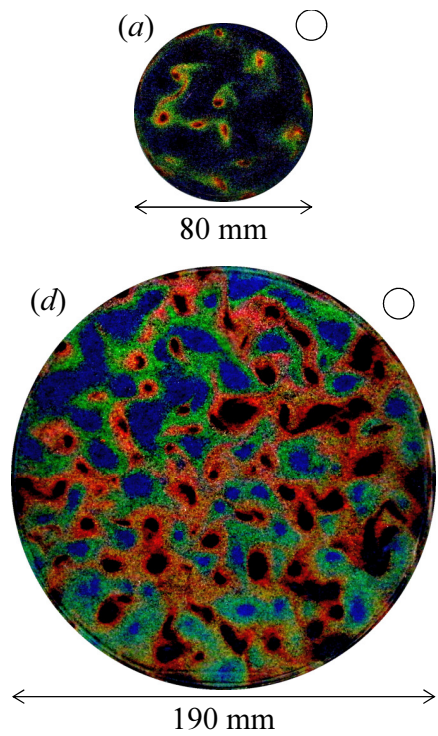

(b)
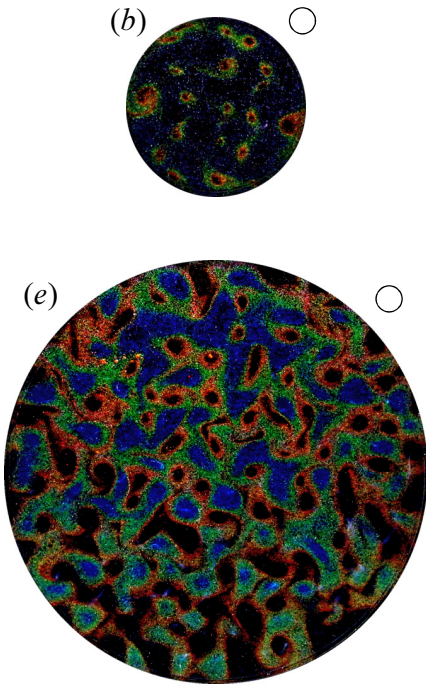

(c)
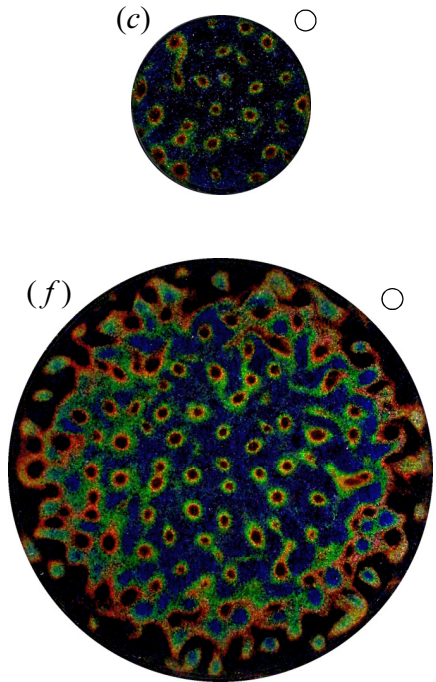

FiguRE 9. Examples of horizontal temperature fields visualized using encapsulated TLCs at $z=0.75 H$ for $(a) T a=1.0 \times 10^{7},(b) 3.0 \times 10^{7}$, and $(c) 1.0 \times 10^{8}$ with $\Gamma=2.00$, and $(d)$ $T a=1.0 \times 10^{7},(e) 3.0 \times 10^{7}$, and $(f) 1.0 \times 10^{8}$ with $\Gamma=4.75$. Open circles appearing on the upper right of each image indicate the horizontal scale of the columnar vortices predicted by Sakai (1997).

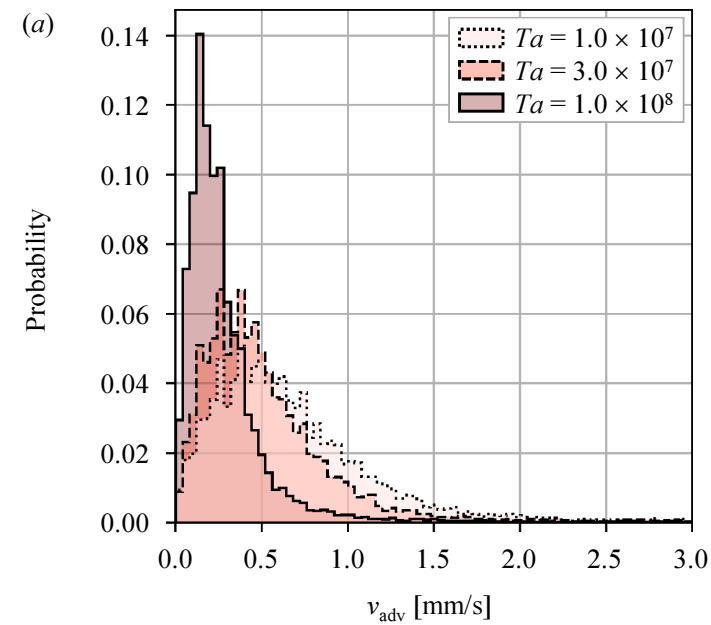

(b)

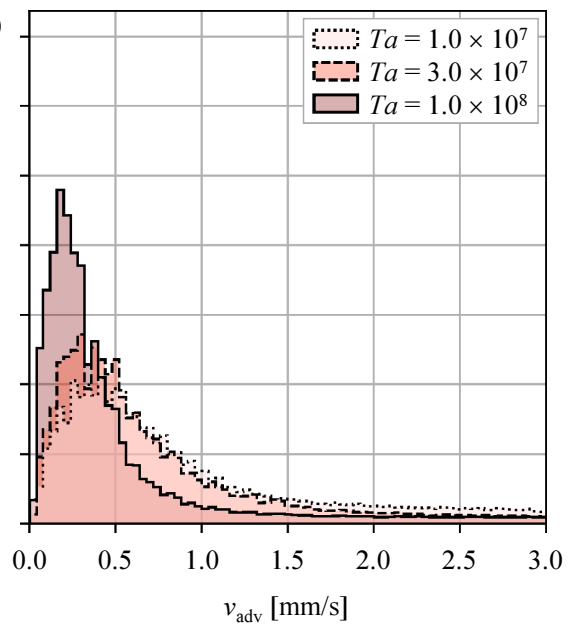

FIgURE 10. Probability distributions of horizontal advection speeds of columnar vortices $v_{\text {adv }}$ for three different values of $T a ;(a) \Gamma=2.00$, and $(b) \Gamma=4.75$.

where $\boldsymbol{r}(t)$ denotes the vortex position vector obtained by the vortex detection method (Noto et al. 2018). The probability distributions of $v_{\text {adv }}$ for all our settings of $T a$ and $\Gamma$ were plotted and analysed (figure 10).

From all the conditions examined, the horizontal translational motion of columnar vortices is extremely slow compared with that of each of the flow fields (figure 10). Specifically, $v_{\text {adv }}$ is $O\left(10^{-1} \mathrm{~mm} / \mathrm{s}\right)$ whereas a typical geostrophic velocity $U_{0}$ is $O\left(10^{0} \mathrm{~mm} / \mathrm{s}\right)$. Moreover, $v_{\text {adv }}$ decreases as $T a$ increases. This result is consistent with a transition from the CTC regime to the cellular regime as $T a$ increases. Basically, the distributions are 
quite similar for vessels of different aspect ratios, except for the highest $T a$ setting with the larger aspect ratio, where the corresponding $F r$ value is close to 0.1. Considering only the difference in the cylinder radii, an obvious difference under this condition may therefore come from the effect of the centrifugal force, even though all the present conditions are satisfying the conventional criterion to disregard any influence of centrifugal forces, $\mathrm{Fr}<0.1$. To confirm this possibility, the probability distribution for columnar vortices present inside the region $r \leqslant 40 \mathrm{~mm}$ for the $\Gamma=4.75$ vessel (corresponding to $\Gamma \leqslant 2.00$ ), is replotted (figure 11 ), here labelled $\Gamma=4.75$ (inside) to separate the effect of centrifugal force. The distribution of $\Gamma=4.75$ (inside) agrees well with that of $\Gamma=2.00$. The probability distribution outside the region $r>40 \mathrm{~mm}$, labelled $\Gamma=4.75$ (outside), shifts slightly towards the higher-speed region compared with that for the inside region. In addition to this separately plotted histogram, the averaged radial components of advection speeds $v_{\text {adv }}$ at each radial position $\left\langle v_{r}(r)\right\rangle$ is examined to understand details of the effect of the centrifugal force; $\left\langle v_{r}(r)\right\rangle$ defined as

$$
\left\langle v_{r}(r)\right\rangle=\frac{1}{N} \sum_{i=1}^{N}\left(v_{x i} \cos \theta_{i}+v_{y i} \sin \theta_{i}\right),
$$

is plotted on figure 12 , where $v_{x}$ and $v_{y}$ are the $x$ and $y$ components of the advection velocity of columnar vortices located at $(r, \theta)$ in polar coordinates. A gradual increase is observed in the larger vessel at $T a=1.0 \times 10^{8}$ (figure 12b) in concert with increases in $r$. Especially in the range of $0 \leqslant r<60 \mathrm{~mm},\left\langle v_{r}(r)\right\rangle$ is linearly proportional to the radial distance $r$ with a slope of 0.0014 according to a least-squares fit in this range. It is noteworthy that the plots with the smaller vessel at $T a=1.0 \times 10^{8}$ fits the same line (slope) as shown in figure $12 a$. Other conditions are everywhere in near $v_{r}=0$ and it is difficult to identify any significant dependence on the radial position, except for the vicinity of the wall. Sudden drops of the velocity in the region corresponding to radially inward movement starts at $r \sim 0.35 L-0.4 L(r \sim 28-32 \mathrm{~mm}$ for $\Gamma=2.00$ and $r \sim 67-76 \mathrm{~mm}$ for $\Gamma=4.75)$ for all $\mathrm{Ta}$ conditions. These originate in repulsion by wall collisions. The standard deviations of each plot are roughly in the range of 0.2 to $0.6 \mathrm{~mm} / \mathrm{s}$, and increase with $T a$ decreases. These results suggest the conclusion that the centrifugal force working on each columnar vortex as a body force may be seen as the main reason for the differences in the distribution for this $T a$ setting, although heuristically the effects of the centrifugal force have been assumed negligibly small when $F r<0.1$ (Cheng et al. 2018). The influence of centrifugal forces on the vortex motion will be discussed in the next section.

An approximate evaluation of the probability density distributions suggests that their shape is similar to a Maxwell distribution assuming a probability density function (PDF) with more frequent low energies (speed) and fewer high energies. If this is reasonably satisfied, translational motion of the columnar vortices can be regarded as diffusive motion, similar to Brownian motion. An attempt to fit of the histograms (figure 10) by the Maxwell distribution,

$$
P_{M}(v)=A v \exp \left(-v^{2} / B\right)
$$

was performed using the least-squares approximation (figure 13). The classical assumptions underpinning this distribution for the particle speed is that the particles, initially move randomly, and form an idealized gas that is spatially homogeneous (independent of their position) and which is isotropic. There are quite large deviations from the fitted curves are seen, especially in the high-speed region. Table 3 lists the fitting constants, $A$ and $B$, and three speeds of vortex advection, here denoted $v_{\mathrm{mp}}, v_{\text {ave }}$, and $v_{\text {rms }}$. They 


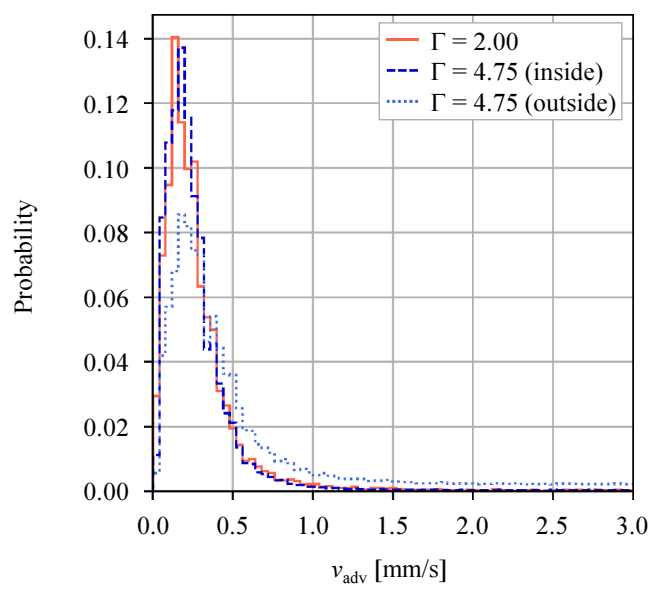

Figure 11. Probability distribution at $T a=1.0 \times 10^{8}$ with $\Gamma=2.00$ (solid line) and separated plots of $\Gamma=4.75$ from different regions; (inside) $r \leqslant 40 \mathrm{~mm}$ (dashed line) and (outside) $r>40 \mathrm{~mm}$ (dotted line).
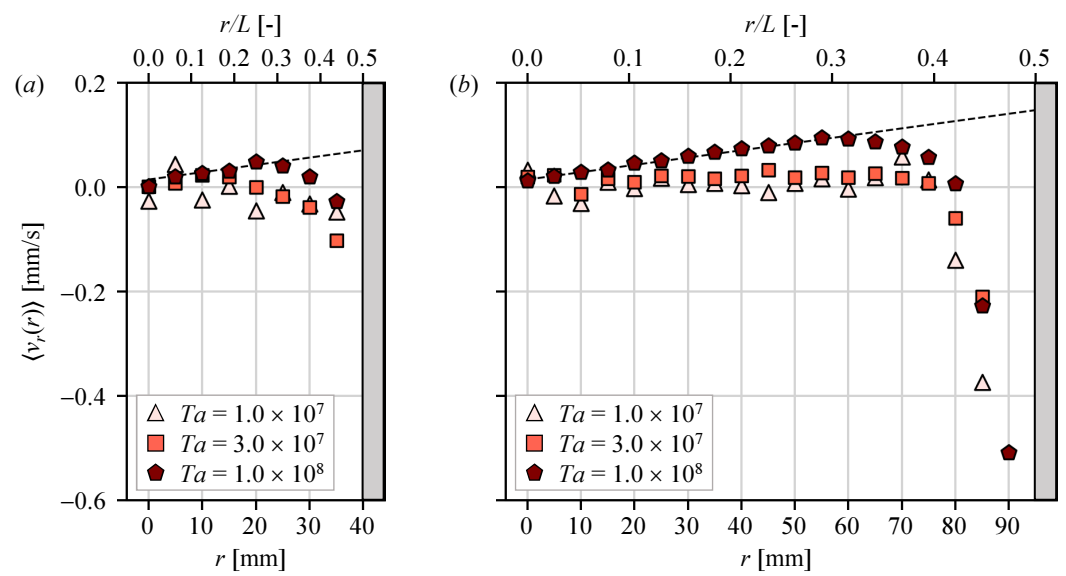

FIGURE 12. Radial position dependence of ensemble averaged radial advection velocity $\left\langle v_{r}\right\rangle ;(a)$ $\Gamma=2.00$, and $(b) \Gamma=4.75$. Each plot is an average of $5 \mathrm{~mm}$ domains. Dashed line drawn in the panels is $\left\langle v_{r}(r)\right\rangle=0.0014 r+0.0143$ obtained by a least-squares fit.

signify respectively the most-probable speed satisfying $d P\left(v_{\mathrm{mp}}\right) / d v=0$, the average speed, and the root-mean-squared speed. For brevity, $v_{\text {adv }}$ is expressed with the $v$ in the above equation. These velocities may also be calculated from the fitted curves; $v_{\mathrm{mp}}$ is given as $v_{\mathrm{mp}}=\sqrt{B}$. It is clear that the speeds depend on $T a$ conditions, although the quality of the fit by the Maxwell distribution with classical assumptions is not perfect. The reasons for the discrepancy will be assessed in the next section and form the main purpose of this paper.

\subsection{Tracking of the multi-timescale behaviour of columnar vortices}

With only the instantaneous observations of the motion of columnar vortices, the deviations from the probability distributions in the Maxwell distributions are difficult to explain. To provide a more detailed description of the vortex motion, a quantification of the differences between actual and idealized particles was performed. The differences arise 


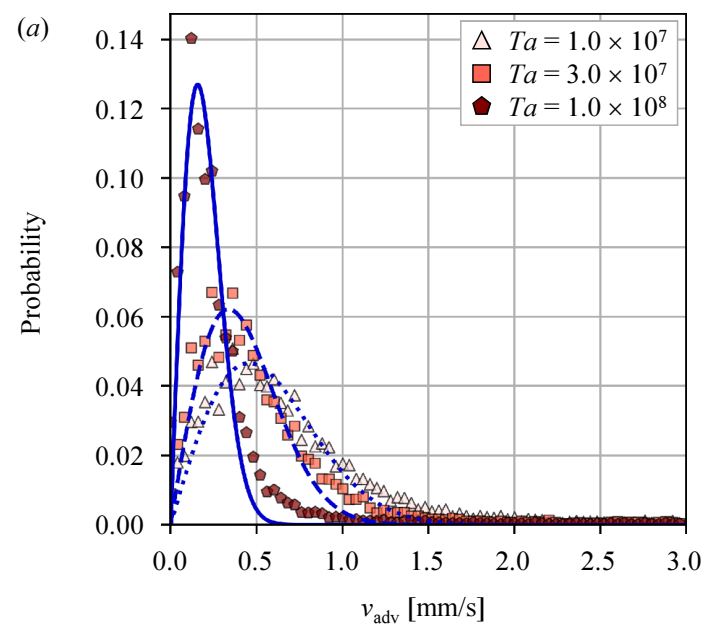

(b)

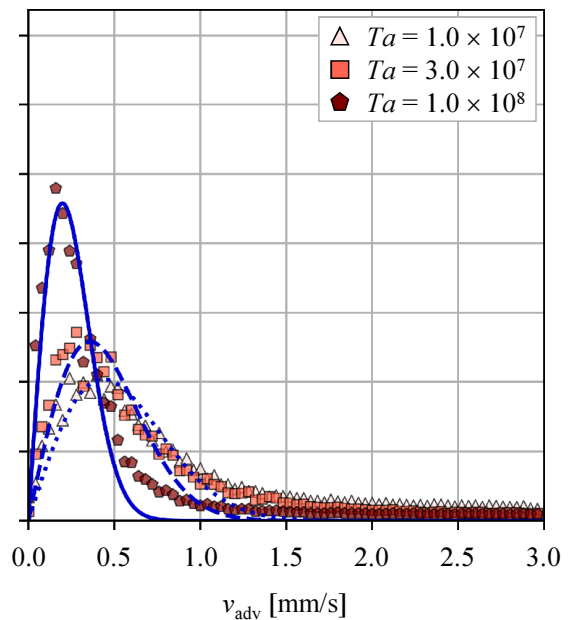

FiguRE 13. PDFs fitted by the Maxwell distribution; the original PDF data are from figure 10 for the three different $T a$ settings with $(a) \Gamma=2.00$, and $(b) \Gamma=4.75$ vessels.

\begin{tabular}{ccccccc}
$\Gamma$ & $T a$ & $A[\mathrm{~s} / \mathrm{mm}]$ & $B\left[\mathrm{~mm}^{2} / \mathrm{s}^{2}\right]$ & $v_{\mathrm{mp}}[\mathrm{mm} / \mathrm{s}]$ & $v_{\text {ave }}[\mathrm{mm} / \mathrm{s}]$ & $v_{\mathrm{rms}}[\mathrm{mm} / \mathrm{s}]$ \\
\hline \hline & $1.0 \times 10^{7}$ & 0.168 & 0.416 & 0.645 & 0.671 & 0.818 \\
2.00 & $3.0 \times 10^{7}$ & 0.303 & 0.229 & 0.478 & 0.532 & 0.675 \\
$1.0 \times 10^{8}$ & 1.326 & 0.0499 & 0.223 & 0.293 & 0.427 \\
\hline & & & & & \\
4.75 & $1.0 \times 10^{7}$ & 0.154 & 0.381 & 0.617 & 0.884 & 1.138 \\
& $3.0 \times 10^{7}$ & 0.237 & 0.257 & 0.507 & 0.718 & 0.947 \\
$1.0 \times 10^{8}$ & 0.760 & 0.0789 & 0.281 & 0.501 & 0.764
\end{tabular}

TABLE 3. Advection speeds of columnar vortices

because of the presence of strong mutual interactions and external (centrifugal) forces. Rather than the analysis of discrete sequences of observations, continuous observations of the vortex motion would serve this purpose. In preparation of this, the lifetime $t_{\text {life }}$ of columnar vortices need to be determined to establish the timescales in which the mutual interactions are appreciable. For the time intervals where an individual vortex is tracked, $t_{\text {life }}$ may also be established (see Noto et al. 2018); in the determination a vortex is deemed to be created at its first detection in a temperature field, and its disappearance through merging defines the end of presence in the series of observations. The probability distribution for $t_{\text {life }}$ during one-hour of observations is then extracted based on this definition (figure 14). Columnar vortices have lifetimes distributed mainly from $10 \mathrm{~s}$ to $100 \mathrm{~s}$ in both vessels here. For $\Gamma=2.00$ in figure $14 a$, there is a clear $T a$ dependence on $t_{\text {life }}$; vortices have shorter lifetimes at lower $T a$ values. With $\Gamma=4.75$ in figure $14 b$, however, the $T a$ dependence is less clear. The reason may be because centrifugal forces are affecting the vortex motion. The sampled number of vortices with the smaller vessel is smaller than that for the larger vessel, however the two plots show similar advection changes, suggesting that the advection may be determined by the advection speed in the finite fluid layer and interactions with the surrounding vortices. The differences along the vertical axis, is suggested to stem from the sampled number of vortices and to be arising 

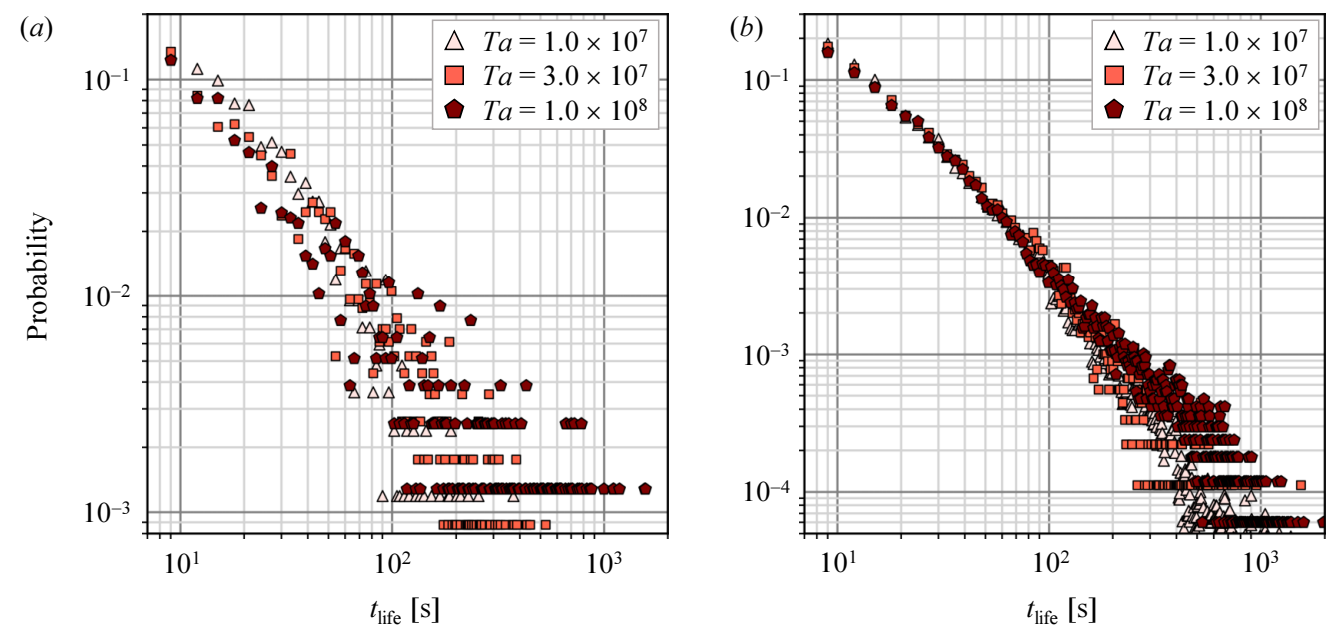

Figure 14. Probability distributions of lifetimes, $t_{\text {life }}$ of columnar vortices for $(a) \Gamma=2.00$, and $(b) \Gamma=4.75$.

from the differences in the aspect ratio. In the present conditions, frequent merging events have been reported in several previous experimental (Boubnov \& Golitsyn 1986; Zhong et al. 1993; Noto et al. 2018) and numerical work (Julien et al. 1996; Pieri et al. 2016) as arising from the absence of sheath structures with vorticities of opposite signs. The historicity, position dependence, and strong mutual interaction within the surroundings may be the cause of the discrepancies appearing in the quality of the fit described in the previous section above. Considering the variations in vortex motion which depend on the observation timescale, vortex lifetimes are very important. This is because the forces affecting the vortices and the heat carried by the vortices are both dependent on the vortex lifetime. From the observed phenomena and the various lifetimes of the vortices, three timescales may be postulated: a short timescale $O\left(10^{0}-10^{1} \mathrm{~s}\right)$ where no vortexvortex interactions occur, an intermediate timescale $O\left(10^{1}-10^{2} \mathrm{~s}\right)$ where strong mutual interactions occur, and a long timescale $O\left(10^{2}-10^{3} \mathrm{~s}\right)$ where the effects of external forces (centrifugal forces) emerge.

To further substantiate this, an analysis of the instantaneous vortex behaviour, as well as vortex tracking by timescales $O\left(>10^{1} \mathrm{~s}\right)$ in which the various forces act on the vortex motion, including their advection history, must be conducted. Noto et al. (2018) reported quasi-regular vortex arrangements obtained in $90 \mathrm{~s}$ periods for larger $T a$ settings, and suggested the possibility of describing vortex motion as an $n$-body problem. Path lines of vortex advection at these timescales (during 30 $\Delta t$ : $90 \mathrm{~s}$ ) were plotted (figure 15). In figure 15, path lines of down-welling columns present in one period (90 s) were cropped and redrawn using colour coding to represent the elapsed time. Depending on the number of vortices, the number of path lines increases with $T a$, and from figure 10 the displacement in one time-step $(3 \mathrm{~s})$ becomes larger as $T a$ decreases. The path lines in figures $15 a, b, d$, and $e$ appear to be random walks, with no directivity. With high $T a$ values in both vessels here (figures $15 c$ and $f$ ), the path lines show weak regularity with the directions of movement restricted by surrounding path lines. As two vortices of opposing vorticity cannot merge (Sprague et al. 2006), this suggests why so few path lines are observed, with the presence of adjacent up-welling vortices, the motion of down-welling vortices tracked here is directional. Further the repulsion from up-welling vortices cannot be ignored in this situation, as Julien et al. (1996) observed when strong 


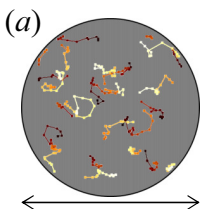

$80 \mathrm{~mm}$

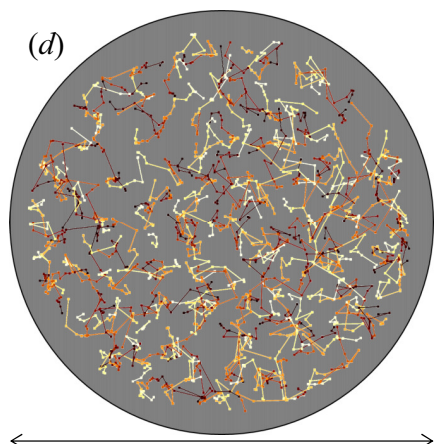

$190 \mathrm{~mm}$ (b)

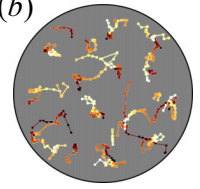

(e)

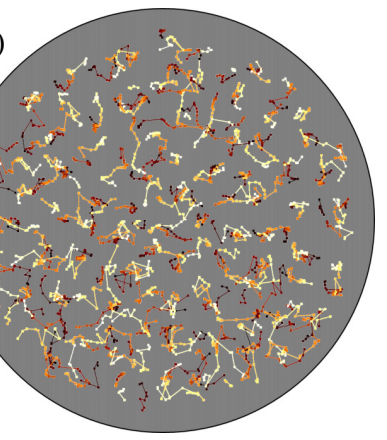

$t+30 \Delta t$ (c)

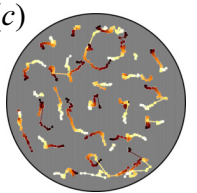

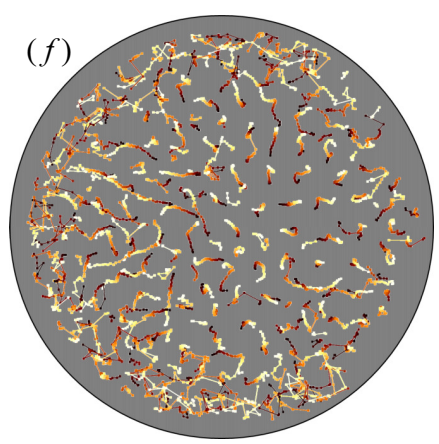

Time $[\mathrm{s}]$

Figure 15. Path lines for advections of the columnar vortices for $90 \mathrm{~s}$; $(a) T a=1.0 \times 10^{7}$, (b) $3.0 \times 10^{7}$, and $(c) 1.0 \times 10^{8}$ with $\Gamma=2.00$, and $(d) T a=1.0 \times 10^{7},(e) 3.0 \times 10^{7}$, and $(f)$ $1.0 \times 10^{8}$ with $\Gamma=4.75$, where the evolution of the path lines is displayed using colour coding. Backgrounds are grey for better visibility.

vortex-vortex interactions were present. The path lines for high $T a$ values may, therefore, be assumed not to represent random motion as shown by Brownian particles.

In addition to the analysis at the intermediate timescales $O\left(10^{1}-10^{2} \mathrm{~s}\right)$, vortex tracking over much longer timescales $O\left(10^{2}-10^{3} \mathrm{~s}\right)$ may be performed simply by using the vortex detection method from the images (Noto et al. 2018). With the larger vessel $(\Gamma=4.75)$ generating centrifugal force effects $(F r \sim 0.1)$ from speedy rotations, such effects appear in the path lines for long times of presence $O\left(10^{2} \mathrm{~s}\right)$ (figure 16), and in figure 16, for clarity, only trajectories longer than $450 \mathrm{~s}$ were plotted. In figure $16 a$, the vortex trajectories appear with repeated jerky movements, in the shape of random walks, and in figure $16 b$ there are numerous trajectories that are largely directed radially outward. This radial motion is not straight, but features a zig-zag pattern as the columns are strongly affected by their surrounding other vortices, as discussed earlier for figure 15 . Columnar vortices in the large-aspect-ratio vessel behave as Brownian particles over short timescales $O\left(10^{0}-10^{1} \mathrm{~s}\right)$, and are affected by centrifugal forces over longer timescales $O\left(10^{2}-10^{3} \mathrm{~s}\right)$. As figure 11 also clearly shows, the influence of centrifugal forces on vortex motion appears only over the long timescales. To explain detailes of this randomwalk-like motion, a further discussion of its statistical nature will be given in the next section.

\section{Statistical analysis of the translational motion}

\subsection{Horizontal diffusive motion of columnar vortices}

Although the probability distributions of vortex advection speeds do not fit a purely Maxwell distribution with its classical assumptions, a random-walk-like motion, however, is observed in the path lines in figures 15 and 16. Based on this, there is the possibility that it will be possible to describe the vortex motion as diffusive motion. Brownian 

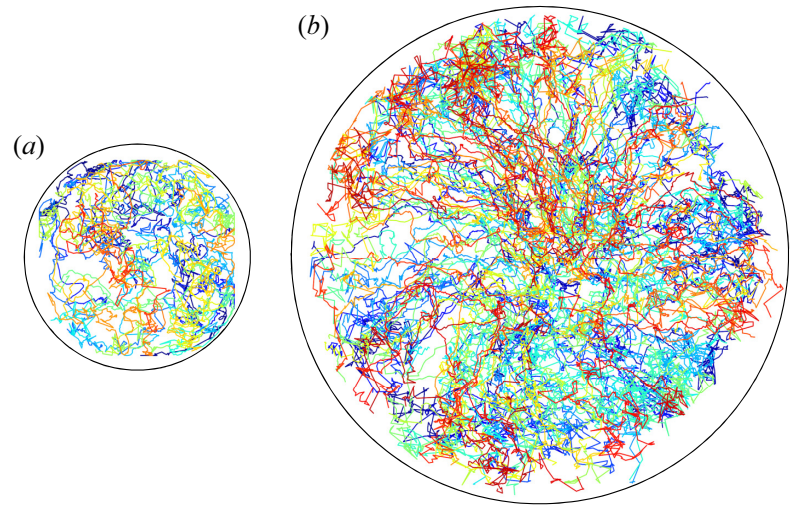

FiguRE 16. Examples of path lines of columnar vortices persisting over $450 \mathrm{~s}$ with $T a=1.0 \times 10^{8} ;(a) \Gamma=2.00$, and $(b) \Gamma=4.75$. Path lines are individually colour coded.

motion (random-walk) shown by molecules in a thermal equilibrium state is one example of commonly occurring normal diffusive motion, natural diffusion without acceleration or deceleration. In other situations of turbulent diffusive arrangements, random Lagrangian particle motion can be represented as diffusive motion arising from macroscopic fluctuations (Solomon et al. 1993). Diffusive motion is characterized by determining the mean squared displacement (MSD),

$$
\operatorname{MSD}=\left\langle\delta \boldsymbol{r}(t)^{2}\right\rangle=\frac{1}{N} \sum_{i=1}^{N}\left[\boldsymbol{r}_{i}(t)-\boldsymbol{r}_{i}(0)\right]^{2},
$$

where $\delta \boldsymbol{r}(t)$ is the displacement from the initial vortex position $\boldsymbol{r}(0)$ with time $t$; angular brackets \langle\rangle indicate an ensemble average. The time dependence of the MSD for normal diffusion processes is given by

$$
\left\langle\delta \boldsymbol{r}(t)^{2}\right\rangle \sim t^{\gamma} \text { with } \gamma=1
$$

Instances of $\gamma \neq 1$ indicate anomalous diffusion: super-diffusion $(1<\gamma<2)$ influenced by acceleration and sub-diffusion $(0<\gamma<1)$ influenced by deceleration. Diffusive motion commonly indicates the motion of tiny molecules (particles). Meuel et al. (2012) performed an MSD analysis to investigate the motion of tropical cyclones, and quantification of diffusive motions of such macroscopic structures are also informative to understand the power sources and timescales dominating the motion. In the present study, an MSD analysis was applied to the horizontal motion of the columnar vortices to be able to discuss the complicated motion presently accepted as diffusive motion; the calculated MSD are plotted in figure 17. To ensure the reliability of the statistics, only ensemble averages with more than 100 vortices are plotted on figure 17, this results in some discrepancy with the lifetimes shown in figure 14. The common feature to note in figure 17 is the relatively large MSD values appearing at low $T a$ settings for both vessels with their different aspect ratios. The magnitude of the MSD reflects the speed of the diffusive motion of the columnar vortices, the speed of movements of the columns moving away from a specific position, the coefficient of diffusion $D(t)$, which can be obtained from

$$
D(t) \equiv \frac{\left\langle\delta \boldsymbol{r}(t)^{2}\right\rangle}{2 \Delta t}
$$

Here, $\Delta t$ is the time interval of the vortex tracking; in the present case, $\Delta t=3 \mathrm{~s}$. For both vessels, lower $T a$ settings show larger $D(t)$ at $O\left(10^{0}-10^{2} \mathrm{~s}\right)$. The calculated 


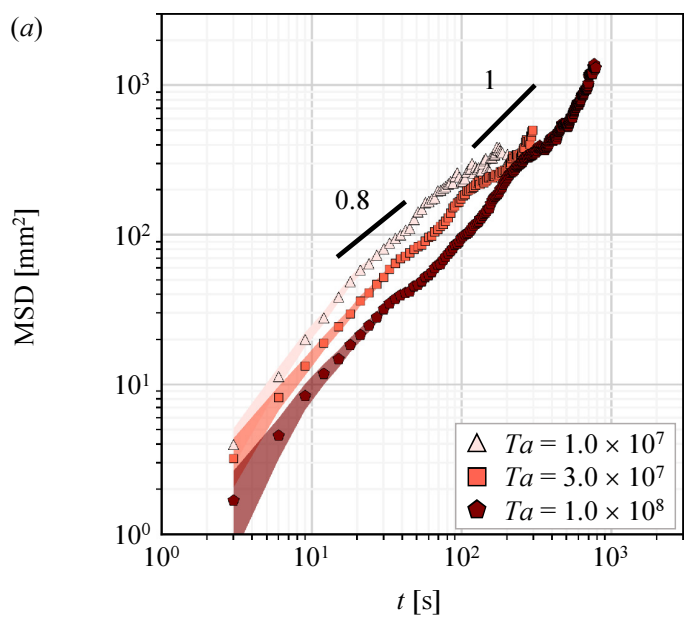

(b)

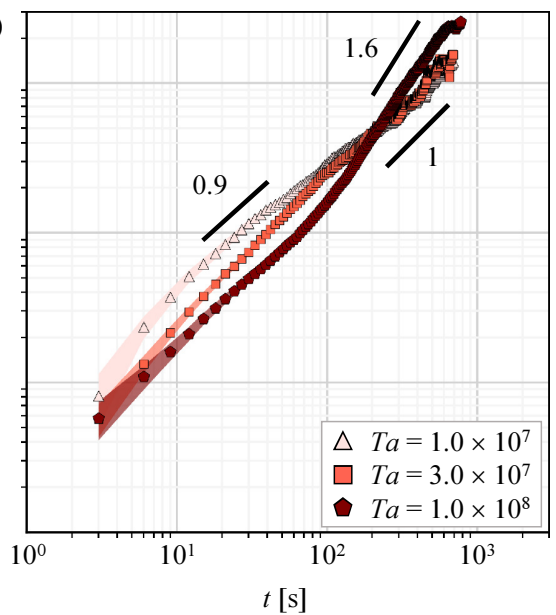

FiguRE 17. Mean squared displacement (MSD) $\left\langle\delta \boldsymbol{r}(t)^{2}\right\rangle$ of the horizontal motion of columnar vortices for different $T a$ values with $(a) \Gamma=2.00$, and $(b) \Gamma=4.75$. The numbers accompanying the black solid lines are the $\gamma$ values. Faded-colour areas behind each plot indicate the standard deviation of the MSD.

$D(t)$ are in the range of $10^{-7}$ to $10^{-6} \mathrm{~m}^{2} / \mathrm{s}$, with the coefficient of viscous diffusion $\nu=1.0 \times 10^{-6} \mathrm{~m}^{2} / \mathrm{s}$, and the coefficient of thermal diffusion $\kappa=1.4 \times 10^{-7} \mathrm{~m}^{2} / \mathrm{s}$. For the vessel with $\Gamma=2.00, D(t)$ decreases as $T a$ increases over the observed period. In addition to this $T a$ dependence of the coefficient of diffusion, time variations in the slopes, $\gamma>1$ in $O\left(10^{0}-10^{1} \mathrm{~s}\right), \gamma<1$ in $O\left(10^{1} \mathrm{~s}\right)$, and $\gamma \geqslant 1$ in $O\left(10^{1}-10^{2} \mathrm{~s}\right)$ are also observed in the smaller vessel. However, for $\Gamma=4.75$, there is no clear $T a$ dependence, especially over long timescales. There are clear differences at the highest $T a$ values in the larger vessel (filled pentagons in figure 17b); the high $T a$ MSD plots cross the two other lower $T a$ conditions because of an abrupt increase in $\gamma$ around $O\left(10^{2} \mathrm{~s}\right)$, from $\gamma \sim 1$ in $O\left(10^{1} \mathrm{~s}\right)$ to $\gamma \sim 1.6$ in over $O\left(10^{2} \mathrm{~s}\right)$. This difference appears only for the highest $T a$ and may be ascribed to the effect of centrifugal forces. Centrifugal force accelerates the vortex motion in the radial direction, making the columnar vortices more easily diffuse and this results in the super-diffusive motion. Further the intermediate timescale $O\left(10^{1} \mathrm{~s}\right)$ shows slight, but gradual decreases in $\gamma$ for all settings. Viewing path lines drawn at the intermediate timescale (for $90 \mathrm{~s}$ ) in figure 15, there is some regularity in the path lines, a result of the mutual interactions between adjacent vortices. This behaviour corresponds to a deceleration of the horizontal motion of the columnar vortices appearing as sub-diffusive motion $\gamma<1$. Further discussion of this is provided below.

\subsection{Anomalous diffusion of columnar vortices}

For the higher $T a$ settings, a smooth MSD plot (figure 17) is obtained over timescales of $O\left(>10^{2} \mathrm{~s}\right)$ as the number of long-lived vortices is large. It is however impossible to obtain smooth curves for the lower $T a$ settings because the lifetimes of the columnar vortices are relatively short making it difficult to record a sufficiently large number of data points for ensemble averaging. With the slope of the MSD for $\gamma \neq 1$, the translational motion of the columnar vortices cannot be considered a pure normal diffusive motion like the random-walk displayed by Brownian particles. In particular, as discussed at the end of the previous section 5.1, intermediate timescales $O\left(10^{1} \mathrm{~s}\right)$ for $\Gamma=2.00$ and $T a=1.0 \times 10^{8}$ show slight decreases in $\gamma$ to $\gamma \sim 0.8-0.9$. For this setting, the vortex motion represented as the path lines in figure 15 is restricted by the adjacent vortices 


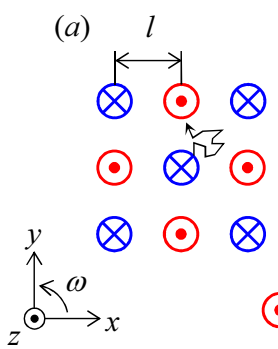

(b)

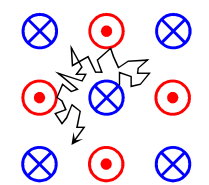

(c)

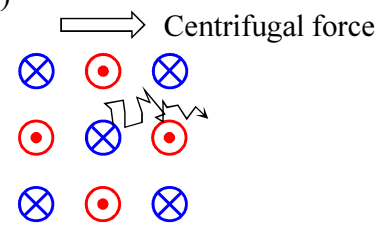

Up-welling Down-welling

Figure 18. Conceptual diagram of the vortex motion without centrifugal force effects $(a)$ $t_{\text {life }}<\tau$ and $(b) t_{\text {life }} \geqslant \tau$, and with centrifugal force effects $(c) t_{\text {life }} \gg \tau$.

\begin{tabular}{ccccc}
$\Gamma$ & $T a$ & $v_{\mathrm{mp}}[\mathrm{mm} / \mathrm{s}]$ & $L_{\text {vortex }}[\mathrm{mm}]$ & $\tau[\mathrm{s}]$ \\
\hline \hline & $1.0 \times 10^{7}$ & 0.645 & 16.5 & 25.6 \\
2.00 & $3.0 \times 10^{7}$ & 0.478 & 11.7 & 24.5 \\
& $1.0 \times 10^{8}$ & 0.223 & 9.34 & 41.9 \\
\hline & & & & \\
4.75 & $1.0 \times 10^{7}$ & 0.617 & 16.5 & 26.7 \\
& $3.0 \times 10^{7}$ & 0.507 & 11.7 & 23.1 \\
$1.0 \times 10^{8}$ & 0.281 & 9.34 & 33.2
\end{tabular}

TABLE 4. Mean free-time for columnar vortices for different $\Gamma$ and $T a$ values.

of opposite vorticities. The two types of down-welling and up-welling vortices, do not merge, but assume a checkerboard (regular grid) pattern in the vortex arrangement, the random-walk-like motion of a down-welling vortex follows the patterns suggested by the arrow in figure $18 a$. This may be interpreted to show that the vortex motion enclosed by a barrier of different-type vortices at this intermediate timescale (figure 18b).

To understand this anomaly in the diffusive vortex motion, the characteristics of columnar vortices different from Lagrangian particles must be considered because the columns are macroscopic flow structures, and are continuous phase to the ambient fluid. For instance, the size of the columnar vortices must be considered unlike negligibly small particles. In addition, columnar vortices have a wide range of lifetimes (observable lives). Depending on the time of observation (lifetime), the observed diffusive motion may vary as strong mutual interactions shown as regularities in figure 15. It may be that the relevant mutual interactions from the surroundings appear at the collision of one vortex with another, making it necessary to consider the mean free-time $\tau$ of the columnar vortices. In this case, $\tau$ is the length of time between the collisions of one vortex and another and is given by $\tau=l / v_{\mathrm{mp}}$, with $l$ the mean free-distance. Assuming that columnar vortices fill the entire fluid layer, $l$ is equivalent to $L_{\mathrm{vortex}}$, and based on this the calculated $\tau$ durations are listed in table 4 .

For $t_{\text {life }}>\tau$, the vortex motion is affected by different adjacent vortices, as they repulse each other. As a result, the repulsions would yield an enclosure effect which slows the vortex motion and prevents unimpeded normal diffusive motion (figure $18 b$ ). The mean free-times $\tau$ listed in table 4 indicate that such an effect appears at $O\left(10^{1} \mathrm{~s}\right)$. From figure 17, the sub-diffusive trend, $\gamma \sim 0.8-0.9$, clearly reflects this effect, and the columnar vortices diffuse little over intermediate timescales.

Under conditions without the effect of centrifugal forces (or even negligibly small 
effects), there is no very large difference in $\tau$ between the two vessels with different aspect ratios here. When centrifugal forces are working (at $T a=1.0 \times 10^{8}$ in $\Gamma=4.75$ ), $\tau$ becomes shorter than that of $\Gamma=2.00$ because the horizontal motion is accelerated by this externally imposed force. The centrifugal forces move the down-welling vortices outward in the radial direction because the density of cold down-welling vortices is larger (figure 18c). The up-welling vortices (lighter than the down-welling) are moved radially inwards in the vessel. Frequent collisions, which oppose free advection of columns towards each other, due to these radial accelerations are the reasons why $\tau$ is shorter for the larger vessel than for the smaller vessel. In addition, for the larger vessel, the columnar vortex in the system undergoes super-diffusive motion due to the centrifugal forces over the long timescales $O\left(>10^{2} \mathrm{~s}\right)$. A large value of $\gamma, \gamma \sim 1.6$, as indicated in figure 17, supports this conclusion even though the mean free-time $\tau$ is shorter when a centrifugal force is working than when it is absent. The large value of $\gamma$ for longer timescales $O\left(>10^{2} \mathrm{~s}\right)$ observed in figure $17 b$ with the highest $T a$ value, would be sustained or further enhanced for vessels of larger horizontal extents, and yield super-diffusive motion as in Lévy flights (e.g., Shlesinger et al. 1993; Solomon et al. 1993), a repetition of stop-go motion. The $\gamma$ asymptotes towards 2 indicating a ballistic diffusion if the experiment could be conducted in a vessel of infinite horizontal extent.

Columnar vortices are not completely isolated from their surroundings but are part of a continuous structure in the surrounding fluid as they are macroscopic flow structures as described above. Therefore, it is impossible to consider these as perfect rigid-body columns of tiny floating particles as discussed above. The unclearly delineated borders and variable body, which are easily affected by their surroundings, are the reasons why clear flight events are not observed. Merging events of the same type of vortices are also thought to be a reason. Commonly, a vortex is surrounded by two types of vortices with a somewhat loose regularity (figure 18). Vortices with opposite vertical flow directions repel each other, whereas the same types of vortices merge (e.g., Zhong et al. 1993; Julien et al. 1996; Pieri et al. 2016; Noto et al. 2018). Ideally, the probability that a vortex collides with either type of vortex is almost even for high $T a$ values, because the population of up-welling and down-welling vortices are almost the same (Vorobieff \& Ecke 2002; Sprague et al. 2006). Opposite-type vortices behave as a barrier preventing normal (random) diffusive motion. The geometric (quasi-polygonal) path lines (figures $15 c$ and $f$ ) are formed by this directivity because of the enclosure effect. Further, merging events do not affect the vortex motion and do not appear in the MSD analysis (figure 17), but only affect the lifetimes (figure 14). These various characteristics of the columnar vortices enable some balance resulting in the $\gamma$ exponent close to unity.

\section{Concluding remarks}

To characterize horizontal translational motion of columnar vortices formed in rotating Rayleigh-Bénard convection, laboratory experiments using water confined in two vessels of different radii were conducted. With different aspect ratios, the influence of the centrifugal forces was investigated while keeping other non-dimensional parameter values fixed. Direct velocity field measurements in vertical cross-sections by particle image velocimetry were made to identify practical rotational parameter values suggesting the formation of prominent columnar vortices by evaluating localized characteristics of the columnar vortices. In the present experimental configuration, columnar vortices formed when the convective Rossby number, $R o<0.4$ ensured vertical coherence of the velocity field. For these settings, experiments tracking of the columnar vortices in horizontal cross-sections were conducted using thermochromic liquid crystal visualization. The 
horizontal motion was tracked periods up to $10^{3} \mathrm{~s}$. The instantaneous profiles of the horizontal advection speeds were difficult to characterize as random-walk-like motion as represented in Maxwell distributions. In observing the temporal motion of the columns, intense mutual interactions, such as merging and repulsion among the vortices appeared as some regularity of vortex path lines, were observed. Further, the effect of centrifugal forces appeared on timescales of $O\left(10^{2} \mathrm{~s}\right)$ even though all the present conditions should in practice be negligible contributions of the centrifugal force $(F r<0.1)$. The mean squared displacement analyses on the horizontal motions of the columnar vortices showed that the motions may be described as anomalous diffusive motion. Presenting a combination of the lifetime of columnar vortices and various other characteristics such as generation, disappearance, merging, and repulsion, the horizontal motion of the columnar vortices were categorized as different diffusive motions: sub-diffusive motion (decelerated advection) due to an enclosure effect arising from the surroundings at $O\left(10^{1}-10^{2} \mathrm{~s}\right)$, and super-diffusive motion (radially accelerated advection) because of centrifugal forces at $O\left(>10^{2} \mathrm{~s}\right)$.

The authors appreciate Dr. Yuzuru Sato and Prof. Keith Julien for fruitful discussions and helpful advices.

\section{REFERENCES}

Abdullah, N., Talib, A. R. A., JaAfar, A. A., Salleh, M. A. M. \& Chonga, W. T. 2010 The basics and issues of thermochromic liquid crystal calibrations. Exp. Therm. Fluid. Sci. 34, 1089-1121.

Aurnou, J. M., Calkins, M. A., Cheng, J. S., Julien, K., King, E. M., Nieves, D., Soderlund, K. M. \& Stellmach, S. 2015 Rotating convective turbulence in earth and planetary cores. Phys. Earth \& Planet. In. 246, 52-71.

Boubnov, B. M. \& Golitsyn, G. S. 1986 Experimental study of convective structures in rotating fluids. J. Fluid Mech. 167, 503-531.

Chandrasekhar, S. 1961 Hydrodynamic and Hydromagnetic Stability. Oxford University Press.

Cheng, J. S., Aurnou, J. M., Julien, K. \& Kunnen, R. P. J. 2018 A heuristic framework for next-generation models of geostrophic convective turbulence. Geophys. \&3 Astro. Fluid $112(4), 277-300$.

Cheng, J. S., Stellmach, S., Ribeiro, A., Grannan, A., King, E. M. \& Aurnou, J. M. 2015 Laboratory-numerical models of rapidly rotating convection in planetary cores. Geophys. J. Int. 201, 1-17.

Grannan, A. M., Favier, B., Le Bars, M. \& Aurnou, J. M. 2016 Tidally forced turbulence in planetary interiors. Geophysical Journal International 208 (3), 1690-1703.

Grooms, I., Julien, K., Weiss, J. B. \& Knobloch, E. 2010 Model of convective Taylor column in rotating Rayleigh-Bénard convection. Phys. Rev. Lett. 104, 224501.

Horn, S. \& Aurnou, J. M. 2018 Regimes of coriolis-centrifugal convection. Phys. Rev. Lett. 120 (20), 204502.

Jones, C. A. 2011 Planetary magnetic fields and fluid dynamos. Annu. Rev. Fluid Mech. 43, 583-614.

Julien, K., Aurnou, J. M., Calkins, M. A., Knobloch, E., Marti, P., Stellmach, S. \& VASIL, G. M. 2016 A nonlinear model for rotationally constrained convection with ekman pumping. J. Fluid Mech. 798, 50-87.

Julien, K., LegG, S., MCWilliams, J. \& Werne, J. 1996 Rapidly rotating turbulent Rayleigh-Bénard convection. J. Fluid Mech. 322, 243-273.

Julien, K., Rubio, A. M., Grooms, I. \& Knobloch, E. 2012 Statistical and physical balances in low rossby number Rayleigh-Bénard convection. Geophys. Astro Fluid 106, 392-428.

Kunnen, R. P. J., Clercx, H. J. H. \& Geurts, B. J. 2010 Vortex statics in turbulent rotating convection. Phys. Rev. E 82 (3), 036306. 
Kunnen, R. P. J., Corre, Y. \& Clercx, H. J. H. 2014 Vortex plume distribution in confined turbulent rotating convection. Europhys. Lett. 104 (5), 54002.

LiU, Y. \& ECKE, R. E. 2009 Heat transport measurements in turbulent rotating RayleighBénard convection. Phys. Rev. E 80, 036314.

Meuel, T., Prado, G., Seychelles, F., Bessafi, M. \& Kellay, H. 2012 Hurricane track forecast cones from fluctuations. Sci. Rep. 2, 446.

Noto, D., Tasaka, Y., Yanagisawa, T., Park, H. J. \& Murai, Y. 2018 Vortex tracking on visualized temperature fields in a rotating Rayleigh-Bénard convection. J. Vis 6, 987-998.

Pieri, A. B., Falasca, F., Hardenberg, J. V. \& Provenzale, A. 2016 Plume dynamics in rotating Rayleigh-Bénard convection. Phys. Lett. A 380, 1363-1367.

Rajaei, H., Kunnen, R. P. J. \& Clercx, H. J. H. 2017 Exploring the geostrophic regime of rapidly rotating convection with experiments. Phys. Fluids 29 (4), 045105.

RAO, Y. \& ZANG, S. 2010 Calibrations and the measurement uncertainty of wide-band liquid crystal thermography. Meas. Sci. Technol. 21, 015105.

SAKAI, S. 1997 The horizontal scale of rotating convection in the geostrophic regime. J. Fluid Mech. 333, 85-95.

Shlesinger, M. F., Zaslavsky, G. M. \& Klafter, J. 1993 Strange kinetics. Nature 363 (6424), 31.

Solomon, T. H., Weeks, E. R. \& Swinney, H. L. 1993 Observation of anomalous diffusion and lévy flights in a two-dimensional rotating flow. Phys. Rev. Lett. 71 (24), 3975.

Sprague, M., Julien, K., Knobloch, E. \& Werne, J. 2006 Numerical simulation of an asymptotically reduced system for rotationally constrained convection. J. Fluid Mech. 551, 141-174.

Stellmach, S., Lischper, M., Julien, K., Vasil, G., Cheng, J. S., Ribeiro, A., King, E. M. \& Aurnou, J. M. 2014 Approaching the asymptotic regime of rapidly rotating convection: Boundary layers versus interior dynamics. Phys. Rev. Lett. 113, 254501.

Stevens, R. J. A. M., Clercx, H. J. H. \& Lohse, D. 2012 Breakdown of the large-scale wind in $\gamma=1 / 2$ rotating Rayleigh-Bénard flow. Phys. Rev. E 86, 056311.

Stevens, R. J. A. M., Clercx, H. J. H. \& Lohse, D. 2013 Heat transport and flow structure in rotating Rayleigh-Bénard convection. Euro. J. Mech. B/Fluids 40, 41-49.

Vorobieff, P. \& Ecke, R. E. 1998 Vortex structure in rotating Rayleigh-Bénard convection. Physica D 123, 153-160.

VorobiefF, P. \& ECKe, R. E. 2002 Turbulent rotating convection: an experimental study. J. Fluid Mech. 458, 191-218.

Weiss, S. \& Ahlers, G. 2011 Heat transport by turbulent rotating Rayleigh-Bénard convection and its dependence on the aspect ratio. J. Fluid Mech. 309, 1-20.

Wiberg, R. \& Lior, N. 2004 Errors in thermochromic liquid crystal thermometry. Rev. Sci. Instrum. 75, 2985.

Zhong, F., Ecke, R. E. \& Steinberg, V. 1993 Rotating Rayleigh-Bénard convection: asymmetric modes and vortex states. J. Fluid Mech. 249, 135-159. 